\title{
On the Stochastic Dynamics of a Social Epidemics Model
}

\author{
Xun-Yang Wang, ${ }^{1,2}$ Peng-Zhan Zhang, ${ }^{1,2}$ and Qing-Shan Yang ${ }^{3}$ \\ ${ }^{1}$ College of Electrical and Information Engineering, Lanzhou University of Technology, Lanzhou, Gansu 730050, China \\ ${ }^{2}$ Department of Applied Mathematics, Lanzhou University of Technology, Lanzhou, Gansu 730050, China \\ ${ }^{3}$ KLAS (Key Laboratory of Applied Statistics of MOE), School of Mathematics and Statistics, Northeast Normal University, \\ Changchun, Jilin 730050, China
}

Correspondence should be addressed to Qing-Shan Yang; yangqr66@gmail.com

Received 15 June 2016; Accepted 4 December 2016; Published 2 January 2017

Academic Editor: Josef Diblík

Copyright (C) 2017 Xun-Yang Wang et al. This is an open access article distributed under the Creative Commons Attribution License, which permits unrestricted use, distribution, and reproduction in any medium, provided the original work is properly cited.

Alcohol abuse is a major social problem, which has caused a lot of damages or hidden dangers to the individual and the society. In this paper, with random factors of alcoholism considered in mortality rate of compartment populations, we formulate a stochastic alcoholism model according to compartment theory of infectious disease. Based on this model, we investigate the long-term stochastic dynamics behaviors of two equilibria of the corresponding deterministic model and point out the effect of random disturbance on the stability of the system. We find that when $R_{0} \leq 1$, we get the estimation between the trajectory of stochastic system and $E_{0}=\left(\Pi / \mu_{s}, 0,0,0\right)$ in the average in time with respect to the disturbance intensity, while when $R_{0}>1$, stochastic system is ergodic and has the unique stationary distribution. Finally, we carry out numerical simulations to support the corresponding theoretical results.

\section{Introduction}

Along with the rapid development of human civilization, the pressure of human life is becoming more and more large, especially in the spirit and emotion. In order to release the pressure, many people will lose their rationality and take some unwise actions; for example, they will take addictive drugs or indulge in long-term alcohol abuse or heavy smoking [1]. These extreme behaviors not only seriously hurt the people's own health but also cause serious security risks to the total society; for example, drunk driving and drunk dangerous sexual behaviors have frequently occurred [2-4]. Sociologists have noticed these undesirable negative phenomena, which are called social diseases with great destructive and infectivity due to mental or habitual addiction [5]. In the above social phenomena, alcoholism is particularly serious, showing the trend of lowering ages and femininity [6].

Actually, similar to some typical infectious diseases, alcohol abuse also has a strong infectiousness, especially for the social crowd in frequent contact; therefore, it is often called social infectious diseases $[5,7]$. In view of this, in the last ten years, many applied mathematicians and interdisciplinary workers are committed to formulating mathematical models to study the spreading behavior of alcohol abuse and predict the trend of alcoholism from a mathematical point of view [8-14].

Sanchez et al. [8] earlier proposed a simple and basic mathematical model with relapse to describe the spread and infection of alcohol abuse. In their model, according to the progress of alcoholism, the population is divided into three compartments, that is, normal persons $S(t)$ who do not drink or drink moderately; the alcoholics $D(t)$, who will infect the people around to become new alcoholics; and the persons who temporarily quit drinking, denoted by $R(t)$. The model in $[8]$ is as follows:

$$
\begin{aligned}
S^{\prime} & =\Lambda-\beta S D-\mu S, \\
D^{\prime} & =\beta S D+\rho R D-(\mu+\phi) D, \\
R^{\prime} & =\phi D-\rho R D-\mu R,
\end{aligned}
$$

based on which, the authors calculated the basic reproduction number of alcoholism $R_{0}$ and proved the existence 
and stability of the two equilibria by virtue of $R_{0}$ and, simultaneously, analyzed the influence of the parameters on the stability of the system. However, this model is somewhat rough and simple. On the basis of this model, many authors have built new mathematical models from different angles to study the behavior of alcoholism. Taking the different stages of alcohol abuse into account, $[9,10]$ proposed a twostage alcoholism model, while [10] also investigated different infection rates according to the two stages. References [12] considered the warning function of information publicity and education on persons against alcohol abuse. Besides the awareness of information, [14] also considered the time delay between the contact and infection during the course of alcohol abuse. To highlight the negative effects of alcohol abuse, [13] proposed a rather complex model to investigate the effect of alcohol on HIV infection.

Due to the fact that alcoholism can be prevented and treated [15], based on an earlier model of alcoholism [8], Wang et al. [16] proposed an SATR-type alcoholism model, and it is presented as follows:

$$
\begin{aligned}
& S^{\prime}=\mu N-\left(1-u_{1}(t)\right) \frac{\beta S A}{N}-\mu S, \\
& A^{\prime}=\left(1-u_{1}(t)\right) \frac{\beta S A}{N}+\xi T-\left(u_{2}(t)+\mu\right) A, \\
& T^{\prime}=u_{2}(t) A-(\mu+\xi+\delta) T, \\
& Q^{\prime}=\delta T-\mu Q,
\end{aligned}
$$

in which the persons in treatment are considered as a population compartment separately and put forward effective measures to control alcohol abuse. Specifically, in model (2), the total population is partitioned into four compartments: $S(t)$, which refers to the persons who never drink or drink moderately without affecting the physical health; $A(t)$, which refers to the persons who drink heavily; $T(t)$, which refers to the persons being treated by taking pills or other medical interventions after alcoholism; $Q(t)$, which refers to the persons who recover from alcoholism after treatment and never drink again.

Recently, Wang et al. [17] continue to consider model (2) with a distributed time delay between contact and infection during the course of alcoholism. Furthermore, in order to make the model have wider applicability in different environments, they generalize the incidence function from standard case to the abstract one.

Actually, in one hand, alcoholism is a social behavior; therefore, it is only infected and transmitted in a limited population. To depict this attribute, we will adopt saturation generating function $\beta S(t) A(t) /(1+\gamma A(t))$ as [18] does. In the other hand, alcoholism will inevitably be affected by many uncertainties, such as emotions and environment. However, the models in the above-mentioned references did not take these random factors into account. In this paper, based on model (2), we will consider the random disturbances of the mortality in all populations due to the effect of alcohol abuse. To do that, we let $\left(\Omega, \mathscr{F},\left\{\mathscr{F}_{t}\right\}_{t \geq 0}, \mathbb{P}\right)$ be a complete probability space with a filtration $\left\{\mathscr{F}_{t}\right\}_{t \geq 0}$ satisfying the usual conditions (i.e., it is increasing and right continuous while $\mathscr{F}_{0}$ contains all $\mathbb{P}$-null sets) and let $B_{i}(t), 1 \leq i \leq 4$ be 4 independent standard Brownian motions. In practice, we usually estimate a parameter by an average value plus an error term. In this case, the parameters $\mu_{s}, \mu_{a}, \mu_{t}$, and $\mu_{q}$ in (2) change to random variables $\widetilde{\mu_{s}}, \widetilde{\mu_{a}}, \widetilde{\mu_{t}}$, and $\widetilde{\mu_{q}}$, respectively, such that

$$
\begin{aligned}
& \widetilde{\mu_{s}}=\mu_{s}+\text { error }_{1}, \\
& \widetilde{\mu_{a}}=\mu_{a}+\text { error }_{2}, \\
& \widetilde{\mu_{t}}=\mu_{t}+\text { error }_{3}, \\
& \widetilde{\mu_{q}}=\mu_{q}+\text { error }_{4} .
\end{aligned}
$$

By the central limit theorem, the error terms may be approximated by normal distributions with mean zero and variance $\sigma_{i}^{2} d t, 1 \leq i \leq 4$, respectively, so we represent them as follows:

$$
\operatorname{error}_{i} d t=\sigma_{i} d B_{i}(t), \quad 1 \leq i \leq 4 \text {. }
$$

With the above crucial factors considered, in this paper, based on model (2), we can get a new stochastic alcoholism dynamic model which is characterized by

$d S(t)$

$$
=\left(\Pi-\frac{\beta S(t) A(t)}{1+\gamma A(t)}-\mu_{s} S(t)\right) d t+\sigma_{1} S(t) d B_{1}(t),
$$

$d A(t)$

$$
\begin{aligned}
= & \left(\frac{\beta S(t) A(t)}{1+\gamma A(t)}-\xi_{1} A(t)+\xi_{2} T(t)-\mu_{a} A(t)\right) d t \\
& +\sigma_{2} A(t) d B_{2}(t),
\end{aligned}
$$

$d T(t)$

$$
\begin{gathered}
=\left(\xi_{1} A(t)-\xi_{2} T(t)-\eta T(t)-\mu_{t} T(t)\right) d t \\
+\sigma_{3} T(t) d B_{3}(t), \\
d Q(t)=\left(\eta T(t)-\mu_{q} Q(t)\right) d t+\sigma_{4} Q(t) d B_{4}(t) .
\end{gathered}
$$

For the parameters in (5) and their explanations, please see Table 1 . We will utilize stochastic analysis method to investigate the dynamic behavior of system (5).

This paper is arranged as follows. In Section 2, we put forward preliminaries including some tools for stochastic analysis and basic properties of deterministic model corresponding to (5) with $\sigma_{i}=0, i=1,2,3,4$. In Section 3, we discuss the existence and uniqueness of the positive solution of (5). In Section 4, we discuss the stochastic stability of alcohol-free equilibrium point $E_{0}$. In Section 5 , we discuss the stochastic stability of the internal alcoholism equilibrium point $E^{*}$. In Section 6, we carry out some simulations to support our theoretical results, In the last section, we give some conclusions to end this paper. 
TABLE 1: The parameters description of model (5).

\begin{tabular}{|c|c|}
\hline Parameter & Description \\
\hline$\mu_{s}, \mu_{a}, \mu_{t}, \mu_{q}$ & $\begin{array}{l}\text { Death rate in } S(t), A(t), T(t), Q(t) \\
\text { accordingly }\end{array}$ \\
\hline$\beta$ & $\begin{array}{l}\text { Transmission coefficient between } S(t) \text { and } \\
A(t)\end{array}$ \\
\hline$\Pi$ & The recruitment constant of population \\
\hline$\gamma$ & Half saturation coefficient \\
\hline$\xi_{1}$ & The ratio of alcoholics in the treatment \\
\hline$\xi_{2}$ & $\begin{array}{l}\text { The ratio of the treatment population who } \\
\text { are failed and return to be alcoholics }\end{array}$ \\
\hline$\eta$ & $\begin{array}{l}\text { The ratio of successfully treated population } \\
\text { and never drink hereafter }\end{array}$ \\
\hline$\sigma_{i}, i=1,2,3,4$ & $\begin{array}{l}\text { Disturbance intensity according to } \\
\mu_{s}, \mu_{a}, \mu_{t}, \mu_{q}, \text { respectively }\end{array}$ \\
\hline$B_{i}(t), i=1,2,3,4$ & $\begin{array}{l}\text { Brown movement followed by } \mu_{s}, \mu_{a}, \mu_{t}, \mu_{q} \text {, } \\
\text { respectively }\end{array}$ \\
\hline
\end{tabular}

\section{Preliminaries}

Now, we give some criteria on the ergodic property. Denote

$$
R_{+}^{l}=\left\{x \in R^{l}: x_{i}>0, \forall 1 \leq i \leq l\right\} .
$$

In general, let $X$ be a regular temporally homogeneous Markov process in $E_{l} \subset R_{+}^{l}$ described by the stochastic differential equation

$$
d X(t)=b(X(t)) d t+\sum_{r=1}^{d} \sigma_{r}(X(t)) d B_{r}(t),
$$

with initial value $X\left(t_{0}\right)=x_{0} \in E_{l}$ and $B_{r}(t), 1 \leq r \leq d$, are standard Brownian motions defined on the above probability space. The diffusion matrix is defined as follows:

$$
\begin{gathered}
A(x)=\left(A_{i j}(x)\right)_{1 \leq i, j \leq l}, \\
A_{i j}(x)=\sum_{r=1}^{d} \sigma_{r}^{i}(x) \sigma_{r}^{j}(x) .
\end{gathered}
$$

Define the differential operator $L$ associated with (7) by

$$
L=\sum_{i=1}^{l} b_{i}(x) \frac{\partial}{\partial x_{i}}+\frac{1}{2} \sum_{i, j=1}^{l} A_{i j}(x) \frac{\partial^{2}}{\partial x_{i} \partial x_{j}} .
$$

If $L$ acts on a function $V \in C^{2,1}\left(E_{l} \times R_{+} ; R\right)$, then

$$
L V(x)=\sum_{i=1}^{l} b_{i}(x) \frac{\partial V}{\partial x_{i}}+\frac{1}{2} \sum_{i, j=1}^{l} A_{i j}(x) \frac{\partial^{2} V}{\partial x_{i} \partial x_{j}},
$$

where $V_{x}=\left(\partial V / \partial x_{1}, \ldots, \partial V / \partial x_{l}\right)$ and $V_{x x}=\left(\partial^{2} V /\right.$ $\left.\partial x_{i} \partial x_{j}\right)_{l \times l}$. By Itô's formula, we have

$$
\begin{aligned}
d V(X(t))= & L V(X(t)) d t \\
& +\sum_{r=1}^{d} V_{x}(X(t)) \sigma_{r}(X(t)) d B_{r}(t) .
\end{aligned}
$$

Lemma 1 (see [19]). We assume that there exists a bounded domain $U \subset E_{l}$ with regular boundary, having the following properties:

(B.1) In the domain $U$ and some neighborhood thereof, the smallest eigenvalue of the diffusion matrix $A(x)$ is bounded away from zero.

(B.2) If $x \in E_{l} \backslash U$, the mean time $\tau$ at which a path issuing from $x$ reaches the set $U$ is finite, and $\sup _{x \in K} E_{x} \tau<\infty$ for every compact subset $K \subset E_{l}$.

Then, the Markov process $X(t)$ has a stationary distribution $v(\cdot)$ with density in $E_{l}$ such that for any Borel set $B \subset E_{l}$

$$
\begin{aligned}
& \lim _{t \rightarrow \infty} P(t, x, B)=v(B), \\
& P_{x}\left\{\lim _{T \rightarrow \infty} \frac{1}{T} \int_{0}^{T} f(x(t)) d t=\int_{E_{l}} f(x) v(d x)\right\}=1,
\end{aligned}
$$

for all $x \in E_{l}$ and $f(x)$ being a function integrable with respect to the probability measure $v$.

Remark 2. (i) The existence of the stationary distribution with density is referred to Theorem 4.1 on page 119 and Lemma 9.4 on page 138 in [19] while the ergodicity and the weak convergence are referred to Theorem 5.1 on page 121 and Theorem 7.1 on page 130 in [19].

(ii) To verify Assumptions (B.1) and (B.2), it suffices to show that there exists a bounded domain $U$ with regular boundary and a nonnegative $C^{2}$-function $V$ such that $A(x)$ is uniformly elliptical in $U$ and, for any $x \in E_{l} \backslash U, L V(x) \leq-C$ for some $C>0$ (see, e.g., [20, page 1163]).

2.1. Basic Properties of Deterministic Model. To compare some results between the deterministic model and stochastic model, firstly, we let $\sigma_{i}=0, i=1,2,3,4$ to get the corresponding deterministic model as follows:

$$
\begin{aligned}
& S^{\prime}(t)=\Pi-\frac{\beta S(t) A(t)}{1+\gamma A(t)}-\mu_{s} S(t), \\
& A^{\prime}(t)=\frac{\beta S(t) A(t)}{1+\gamma A(t)}-\xi_{1} A(t)+\xi_{2} T(t)-\mu_{a} A(t), \\
& T^{\prime}(t)=\xi_{1} A(t)-\xi_{2} T(t)-\eta T(t)-\mu_{t} T(t), \\
& Q^{\prime}(t)=\eta T(t)-\mu_{q} Q(t) .
\end{aligned}
$$

We can derived the alcohol-free equilibrium $E_{0}$ of the deterministic model corresponding to (13); that is, $E_{0}=$ $\left(\Pi / \mu_{s}, 0,0,0\right)$. By using the method of next generation matrix [21], we can calculate to get the fundamental reproduction number of alcoholism which is

$$
R_{0}=\beta \frac{\Pi}{\mu_{s}} \frac{\xi_{2}+\eta+\mu_{t}}{\left[\xi_{1}\left(\eta+\mu_{t}\right)+\mu_{a}\left(\xi_{2}+\eta+\mu_{t}\right)\right]} .
$$

The biological meaning of $R_{0}$ is as follows. Once an alcoholic is placed in the environment full of healthy persons with the initial population $\Pi / \mu_{s}$, during the course of alcohol 
transmission time $1 /\left(\xi_{1}+\mu_{a}\right)$, the alcoholic will infect the healthy persons. $R_{0}$ denotes the number of healthy persons who are successfully infected.

Denoting $N(t)=S(t)+A(t)+T(t)+Q(t)$ and adding up the four equations in (13), we can get

$$
N^{\prime}(t)=\Pi-\mu_{s} S-\mu_{a} A-\mu_{t} T-\mu_{q} Q \leq \Pi-\mu N(t),
$$

where $\mu=\min \left\{\mu_{s}, \mu_{a}, \mu_{t}, \mu_{q}\right\}$. Obviously, as $t \rightarrow \infty, N(t) \leq$ $\Pi / \mu$. Hence, all the solutions of (13) will fall in

$$
\Omega=\left\{(S, A, T, Q) \in R_{+}^{4} \mid S+A+T+Q \leq \frac{\Pi}{\mu}\right\},
$$

which is easily proven to be an invariant set. Based on the analysis of [16], we list the relevant results of (13) as follows.

Theorem 3. For system (13), when $R_{0}<1$, only an alcohol-free equilibrium $E_{0}=\left(\Pi / \mu_{s}, 0,0,0\right)$ exists and it is globally asymptotically stable; when $R_{0}>1$, besides $E_{0}$ (it is not stable), there exists an internal equilibrium $E^{*}=\left(S^{*}, A^{*}, T^{*}, Q^{*}\right)$, which is globally asymptotically stable.

\section{The Existence and Uniqueness of the Positive Solution of (5)}

For the sake of biological and practical meanings, in this section, we will investigate the long-term behaviors of system (5); that is, no matter how much the disturbance intensity is, it has a unique global positive solution. According to classical theory of the stochastic differential equations, in order to make sure the existence and uniqueness of the global solution of system (5) are under the given initial conditions (i.e., it will not blow up in a limited time), we generally require that the coefficient of stochastic system (5) satisfies the linear growth condition and the local Lipschitz condition [22]. However, the coefficient of system (5) does not satisfy the linear growth condition; therefore, the solution is likely to blow up in a finite time. In the following, we will use the analysis method of Lyapunov functional to prove global existence of positive solution.

Theorem 4. For arbitrary initial value $(S(0), A(0), T(0)$, $\mathrm{Q}(0)) \in R_{+}^{4}$, as $t \geq 0$, system (5) shows a unique positive solution $(S(t), A(t), T(t), Q(t))$, which fall in $R_{+}^{4}$ almost with probability 1; that is, $(S(t), A(t), T(t), Q(t)) \in R_{+}^{4}$, a.s.

Proof. We firstly prove that system (5) shows a unique local solution. Noticing that the right hand of system (5) does not satisfy the local Lipschitz condition, we introduce variable transformations $S=e^{u}, A=e^{v}$; thus, system (5) can be converted into the one whose coefficients satisfy the local Lipschitz condition.

Next, we prove that the only solution is positive and nonexplosive. We assume that system (5) only shows the unique local solution $(S(t), A(t), T(t), Q(t)), t \in\left[0, \tau_{e}\right)$, where $\tau_{e}$ means the explosive time. To show that the solution is global, we should prove $\tau_{e}=\infty$, a.e. There exists a sufficient large $m_{0}$ such that $(S(0), A(0), T(0), Q(0)) \in\left[1 / m_{0}, m_{0}\right]$. For arbitrary positive integer $m \geq m_{0}$, we define the stopping time

$$
\begin{aligned}
\tau_{m} & =\inf \left\{t \in\left[0, \tau_{e}\right): \min \{S(t), A(t), T(t), Q(t)\}\right. \\
& \left.\leq \frac{1}{m} \text { or } \max \{S(t), A(t), T(t), Q(t)\} \geq m\right\},
\end{aligned}
$$

where inf $\phi=\infty$ ( $\phi$ means empty set). Obviously, as $m \rightarrow \infty$, $\tau_{m}$ is monotonically increasing. Due to $\tau_{m} \leq \tau_{e}$, sequence $\left\{\tau_{m}\right\}$ is convergent. Let $\tau_{\infty}=\lim _{m \rightarrow \infty} \tau_{m}$, so $\tau_{\infty} \leq \tau_{e}$ a.s. If we can show $\tau_{\infty}=\infty$, so $\tau_{e}=\infty$ is obviously established.

We take apagoge to prove this conclusion. Assume $\tau_{\infty} \neq$ $\infty$ (i.e., $\tau_{\infty}<\infty$ ); then there must be a large enough positive constant $T$ and a small enough positive constant $\epsilon \in(0,1)$ such that

$$
P\left\{\tau_{\infty} \leq T\right\}>\epsilon
$$

Hence, there exists a positive integer $m_{1} \geq m_{0}$ such that

$$
P\left\{\tau_{m} \leq T\right\} \geq \epsilon, \quad \forall m \geq m_{1}
$$

We define a Lyapunov functional $V: R_{+}^{4} \rightarrow R_{+}$as follows:

$$
\begin{aligned}
V(S, A, T, Q)= & (S-1-\ln S)+(A-1-\ln A) \\
& +(T-1-\ln T)+(Q-1-\ln Q) .
\end{aligned}
$$

By the simple inequality of $u-1-\ln u \geq 0, \forall u>0$, it is easy to know that $V(S, A, T, Q)$ is nonnegative. In virtue of Itô formula, we calculate the stochastic differential of $V(S, A, T, Q)$ along with (5) as follows:

$$
\begin{aligned}
& d V=\left(1-\frac{1}{S}\right) d S+\frac{1}{2 S^{2}}(d S)^{2}+\left(1-\frac{1}{A}\right) d A \\
& +\frac{1}{2 A^{2}}(d A)^{2}+\left(1-\frac{1}{T}\right) d T+\frac{1}{2 T^{2}}(d T)^{2}+(1 \\
& \left.-\frac{1}{Q}\right) d Q+\frac{1}{2 Q^{2}}(d Q)^{2}=\left[\Pi-\mu_{s} S-\frac{\Pi}{S}\right. \\
& +\frac{\beta A}{1+\gamma A}+\mu_{s}+\frac{\sigma_{1}^{2}}{2}-\mu_{a} A-\frac{\beta S}{1+\gamma A}+\xi_{1}-\xi_{2} \frac{T}{A} \\
& +\mu_{a}+\frac{\sigma_{2}^{2}}{2}-\mu_{t} T-\xi_{1} \frac{A}{T}+\xi_{2}+\eta+\mu_{t}+\frac{\sigma_{3}^{2}}{2}-\mu_{q} Q \\
& \left.-\eta \frac{T}{Q}+\mu_{q}+\frac{\sigma_{4}^{2}}{2}\right] d t+\left(1-\frac{1}{S}\right) \sigma_{1} S d B_{1}+(1 \\
& \left.-\frac{1}{A}\right) \sigma_{2} A d B_{2}+\left(1-\frac{1}{T}\right) \sigma_{3} T d B_{3}+\left(1-\frac{1}{Q}\right) \\
& \cdot \sigma_{4} Q d B_{4} \leq\left[\left(1+\frac{1}{\mu}\right) \Pi+\frac{\beta}{\gamma}+\mu_{s}+\mu_{a}+\mu_{t}+\mu_{q}\right.
\end{aligned}
$$




$$
\begin{aligned}
& \left.+\xi_{1}+\xi_{2}+\eta+\frac{\sigma_{1}^{2}+\sigma_{2}^{2}+\sigma_{3}^{2}+\sigma_{4}^{2}}{2}\right] d t+\sigma_{1}(1 \\
& \left.-\frac{1}{S}\right) S d B_{1}+\sigma_{2}\left(1-\frac{1}{A}\right) A d B_{2}+\sigma_{3}\left(1-\frac{1}{T}\right) \\
& \cdot T d B_{3}+\sigma_{4}\left(1-\frac{1}{Q}\right) Q d B_{4}:=L V d t+\sigma_{1}\left(1-\frac{1}{S}\right) \\
& \cdot S d B_{1}+\sigma_{2}\left(1-\frac{1}{A}\right) A d B_{2}+\sigma_{3}\left(1-\frac{1}{T}\right) T d B_{3} \\
& +\sigma_{4}\left(1-\frac{1}{Q}\right) Q d B_{4},
\end{aligned}
$$

where

$$
\begin{aligned}
L V= & \left(1+\frac{1}{\mu}\right) \Pi+\frac{\beta}{\gamma}+\mu_{s}+\mu_{a}+\mu_{t}+\mu_{q}+\xi_{1}+\xi_{2} \\
& +\eta+\frac{\sigma_{1}^{2}+\sigma_{2}^{2}+\sigma_{3}^{2}+\sigma_{4}^{2}}{2}:=K .
\end{aligned}
$$

Integrating the two sides of $d V$ above from 0 to $\tau_{m} \wedge T$, we get

$$
\begin{aligned}
& \int_{0}^{\tau_{m} \wedge T} d V(S(l), A(l), T(l), Q(l)) \leq \int_{0}^{\tau_{m} \wedge T} K d l \\
& +\int_{0}^{\tau_{m} \wedge T}\left[\sigma_{1}\left(1-\frac{1}{S(l)}\right) S(l) d B_{1}(l)\right. \\
& +\sigma_{2}\left(1-\frac{1}{A(l)}\right) A(l) d B_{2}(l) \\
& +\sigma_{3}\left(1-\frac{1}{T(l)}\right) T(l) d B_{3}(l) \\
& \left.+\sigma_{4}\left(1-\frac{1}{Q(l)}\right) Q(l) d B_{4}(l)\right] .
\end{aligned}
$$

Take expectation of the two sides in the above expression to get

$$
\begin{aligned}
& E\left[V\left(S\left(\tau_{m} \wedge T\right), A\left(\tau_{m} \wedge T\right), T\left(\tau_{m} \wedge T\right), Q\left(\tau_{m} \wedge T\right)\right)\right] \\
& \quad \leq V(S(0), A(0), T(0), Q(0))+E \int_{0}^{\tau_{m} \wedge T} K d l \\
& \quad \leq V(S(0), A(0), T(0), Q(0))+K T .
\end{aligned}
$$

Let $\Omega_{m}=\left\{\tau_{m} \leq T\right\}$; there exists $m_{1}>0$, for $\forall m>m_{1}$; by (19), we know $P\left(\Omega_{m}\right) \geq \epsilon . \forall \omega \in \Omega_{m}$, at least one of $S\left(\tau_{m}\right), A\left(\tau_{m}\right), T\left(\tau_{m}\right)$ and $\left(\tau_{m}\right)$ equals $m$ or $1 / m$. Specifically, when $S\left(\tau_{m}, \omega\right)=m$ or $1 / m, A\left(\tau_{m}, \omega\right)=m$ or $1 / m, T\left(\tau_{m}, \omega\right)=$ $m$ or $1 / m$, or $Q\left(\tau_{m}, \omega\right)=m$ or $1 / m$, there will always be

$$
\begin{aligned}
& V\left(S\left(\tau_{m} \wedge T\right), A\left(\tau_{m} \wedge T\right), T\left(\tau_{m} \wedge T\right), Q\left(\tau_{m} \wedge T\right)\right) \\
& \geq(m-1-\ln m) \wedge\left(\frac{1}{m}-1-\ln \frac{1}{m}\right) .
\end{aligned}
$$

Comprehensive consideration of (19) and (24), we can get

$$
\begin{aligned}
& V(S(0), A(0), T(0), Q(0))+K T \\
& \quad \geq E\left[1 _ { \Omega _ { m } ( \omega ) } V \left(S\left(\tau_{m} \wedge T\right), A\left(\tau_{m} \wedge T\right), T\left(\tau_{m} \wedge T\right),\right.\right. \\
& \left.\left.\quad Q\left(\tau_{m} \wedge T\right)\right)\right] \geq \epsilon\left[( m - 1 - \operatorname { l n } m ) \wedge \left(\frac{1}{m}-1\right.\right. \\
& \left.\left.\quad-\ln \frac{1}{m}\right)\right],
\end{aligned}
$$

where $1_{\Omega_{m}}$ denotes the indicator function of $\Omega_{m}$. By letting $m \rightarrow \infty$, it is easy to get the following contradiction:

$$
\infty>V(S(0), A(0), T(0), Q(0))+K T=\infty .
$$

Thus, $\tau_{\infty}=\infty$ a.e. The proof is complete.

Remark 5. This theorem implies that, besides the alcohol free equilibrium, system (5) will always show a unique positive solution, no matter how much the disturbance intensity is.

\section{Stochastic Stability of Alcohol-Free Equilibrium Point $E_{0}$}

Seen from Theorem 4, for deterministic system (13), as $R_{0}<$ 1 , alcohol-free equilibrium point $E_{0}=\left(\Pi / \mu_{s}, 0,0,0\right)$ is globally asymptotically stable, which means the alcohol population will disappear in the appropriate conditions finally, and the alcohol behavior will be effectively controlled. However, there is not any equilibrium point in the stochastic system (5); therefore, in this section, we will discuss the disturbance behavior of system (5) by investigating the stability and the ergodic property of $E_{0}$.

Theorem 6. Assume ( $S(t), A(t), T(t), Q(t))$ is the solution of system (5) with the initial value $(S(0), A(0), T(0), Q(0)) \in R_{+}^{4}$. If

$$
R_{0}=\beta \frac{\Pi}{\mu_{s}} \cdot \frac{\xi_{2}+\eta+\mu_{t}}{\left[\xi_{1}\left(\eta+\mu_{t}\right)+\mu_{a}\left(\xi_{2}+\eta+\mu_{t}\right)\right]} \leq 1,
$$

then

$$
\begin{aligned}
& \limsup _{T \rightarrow \infty} \frac{1}{T} \int_{0}^{T}\left[\left(\mu_{s}-c_{1}\right)\left(S(t)-S_{0}\right)^{2}\right. \\
& \quad+\left(\frac{\mu_{a}}{2} A^{2}-c_{2}\right) A^{2}(t) \\
& \left.\quad+\left(\frac{\eta+\mu_{t}}{2}-c_{3}\right) T^{2}(t)\right] d t \leq c_{1} S_{0}^{2},
\end{aligned}
$$

where $c_{i}, i=1,2,3$ are defined as follows:

$$
\begin{aligned}
& c_{1}=\left[\frac{1}{2 \mu_{s}}\left(\frac{\left(\mu_{s}+\mu_{a}\right)^{2}}{2 \mu_{a}}+\frac{\left(\mu_{s}+\eta+\mu_{t}\right)^{2}}{2\left(\eta+\mu_{t}\right)}\right)+\frac{1}{2}\right] \sigma_{1}^{2}, \\
& c_{2}=\frac{\sigma_{2}^{2}}{2}, \\
& c_{3}=\left(\frac{1}{2}+\frac{\mu_{a}+\eta+\mu_{t}}{2 \xi_{1}}\right) \sigma_{3}^{2} .
\end{aligned}
$$


Proof. Define nonnegative $C^{2}$-function $V_{1}: R_{+} \rightarrow R_{+}$as

$$
V_{1}(S)=\frac{\left(S-S_{0}\right)^{2}}{2} ;
$$

then

$$
\begin{aligned}
d V_{1}= & \left(S-S_{0}\right) d S+\frac{1}{2}(d S)^{2} \\
= & \left(S-S_{0}\right)\left[\left(\Pi-\frac{\beta S A}{1+\gamma A}-\mu_{s} S\right) d t+\sigma_{1} S d B_{1}(t)\right] \\
& +\frac{1}{2}\left(\sigma_{1} S\right)^{2} d t .
\end{aligned}
$$

Hence,

$$
\begin{aligned}
L V_{1} & =\left(S-S_{0}\right)\left(\Pi-\frac{\beta S A}{1+\gamma A}-\mu_{s} S\right)+\frac{1}{2}\left(\sigma_{1} S\right)^{2} \\
& =\left(S-S_{0}\right)\left(\mu_{s} S_{0}-\frac{\beta S A}{1+\gamma A}-\mu_{s} S\right)+\frac{1}{2}\left(\sigma_{1} S\right)^{2} \\
& \leq-\mu_{s}\left(S-S_{0}\right)^{2}-\frac{\beta S_{0}\left(S-S_{0}\right) A}{1+\gamma A}+\frac{1}{2}\left(\sigma_{1} S\right)^{2} .
\end{aligned}
$$

Let

$$
V_{2}(A, T)=A+\frac{\xi_{2}}{\eta+\mu_{t}+\xi_{2}} T
$$

then

$$
\begin{aligned}
d V_{2}= & d A+\frac{\xi_{2}}{\eta+\mu_{t}+\xi_{2}} d T \\
= & \left(\frac{\beta S A}{1+\gamma A}-\xi_{1} A+\xi_{2} T-\mu_{a} A\right) d t+\sigma_{2} A d B_{2}(t) \\
& +\frac{\xi_{2}}{\eta+\mu_{t}+\xi_{2}}\left(\xi_{1} A-\xi_{2} T-\eta T-\mu_{t} T\right) d t \\
& +\sigma_{3} T d B_{3}(t) .
\end{aligned}
$$

Hence,

$$
\begin{aligned}
L V_{2}= & \frac{\beta S A}{1+\gamma A}-\xi_{1} A+\xi_{2} T-\mu_{a} A \\
& +\frac{\xi_{2}}{\eta+\mu_{t}+\xi_{2}}\left(\xi_{1} A-\xi_{2} T-\eta T-\mu_{t} T\right) \\
= & \frac{\beta\left(S-S_{0}\right) A}{1+\gamma A}+\frac{\beta S_{0} A}{1+\gamma A}-\left(\xi_{1}+\mu_{a}\right) A \\
& +\frac{\xi_{1} \xi_{2} A}{\eta+\mu_{t}+\xi_{2}} \\
= & \frac{\beta\left(S-S_{0}\right) A}{1+\gamma A} \\
& +A\left[\frac{\beta S_{0}}{1+\gamma A}-\left(\xi_{1}+\mu_{a}-\frac{\xi_{1} \xi_{2}}{\eta+\mu_{t}+\xi_{2}}\right)\right]
\end{aligned}
$$

$$
\begin{aligned}
& \leq \frac{\beta\left(S-S_{0}\right) A}{1+\gamma A}+A\left[\beta S_{0}-\frac{\xi_{1}\left(\eta+\mu_{t}\right)}{\eta+\mu_{t}+\xi_{2}}-\mu_{a}\right] \\
& \leq \frac{\beta\left(S-S_{0}\right) A}{1+\gamma A} .
\end{aligned}
$$

The last inequality is attributed to the following relation:

$$
\begin{aligned}
& \beta S_{0}-\frac{\xi_{1}\left(\eta+\mu_{t}\right)}{\eta+\mu_{t}+\xi_{2}}-\mu_{a} \leq 0 \Longleftrightarrow \\
& R_{0}=\beta \frac{\Pi}{\mu_{s}} \cdot \frac{\xi_{2}+\eta+\mu_{t}}{\left[\xi_{1}\left(\eta+\mu_{t}\right)+\mu_{a}\left(\xi_{2}+\eta+\mu_{t}\right)\right]} \leq 1 .
\end{aligned}
$$

Consider linear combination $V_{1}+S_{0} V_{2}$; then

$$
L\left(V_{1}+S_{0} V_{2}\right) \leq-\mu_{s}\left(S-S_{0}\right)^{2}+\frac{1}{2}\left(\sigma_{1} S\right)^{2} .
$$

Let

$$
V_{3}=\frac{T^{2}}{2}
$$

then

$$
\begin{aligned}
d V_{3}= & T d T+\frac{1}{2}(d T)^{2} \\
= & T\left(\xi_{1} A-\xi_{2} T-\eta T-\mu_{t} T\right) d t+\sigma_{3} T d B_{3}(t) \\
& +\frac{1}{2}\left(\sigma_{3} T\right)^{2} d t
\end{aligned}
$$

$$
\begin{aligned}
L V_{3} & =T\left(\xi_{1} A-\xi_{2} T-\eta T-\mu_{t} T\right)+\frac{1}{2}\left(\sigma_{3} T\right)^{2} \\
& =\xi_{1} A T-\left(\eta+\mu_{t}+\xi_{2}\right) T^{2}+\frac{1}{2}\left(\sigma_{3} T\right)^{2} .
\end{aligned}
$$

Let

$$
V_{4}=\frac{\left(S-S_{0}+A+T\right)^{2}}{2} ;
$$

then

$$
\begin{aligned}
d V_{4} & =\left(S-S_{0}+A+T\right)(d S+d A+d T) \\
& +\frac{(d S)^{2}+(d A)^{2}+(d T)^{2}}{2}=\left(S-S_{0}+A+T\right) \\
\cdot & {\left[\left(\Pi-\mu_{s} S-\mu_{a} A-\mu_{t} T-\eta T\right) d t+\sigma_{1} S d B_{1}(t)\right.} \\
& \left.+\sigma_{2} A d B_{2}(t)+\sigma_{3} T d B_{3}(t)\right] \\
& +\frac{\left(\sigma_{1} S\right)^{2}+\left(\sigma_{2} A\right)^{2}+\left(\sigma_{3} T\right)^{2}}{2} d t, \\
L V_{4} & =\left(S-S_{0}+A+T\right)\left(\Pi-\mu_{s} S-\mu_{a} A-\mu_{t} T-\eta T\right) \\
& +\frac{\left(\sigma_{1} S\right)^{2}+\left(\sigma_{2} A\right)^{2}+\left(\sigma_{3} T\right)^{2}}{2}=-\mu_{s}\left(S-S_{0}\right)^{2} \\
& -\mu_{a} A^{2}-\left(\eta+\mu_{t}\right) T^{2}-\left(\mu_{s}+\mu_{a}\right)\left(S-S_{0}\right) A-\left(\mu_{s}\right. \\
& \left.+\eta+\mu_{t}\right)\left(S-S_{0}\right) T-\left(\mu_{a}+\eta+\mu_{t}\right) A T
\end{aligned}
$$




$$
\begin{aligned}
& +\frac{\left(\sigma_{1} S\right)^{2}+\left(\sigma_{2} A\right)^{2}+\left(\sigma_{3} T\right)^{2}}{2} \leq-\mu_{s}\left(S-S_{0}\right)^{2}-\frac{\mu_{a}}{2} \\
& \cdot A^{2}-\frac{\left(\eta+\mu_{t}\right)}{2} T^{2}+\frac{\left(\mu_{s}+\mu_{a}\right)^{2}}{2 \mu_{a}}\left(S-S_{0}\right)^{2} \\
& +\frac{\left(\mu_{s}+\eta+\mu_{t}\right)^{2}}{2\left(\eta+\mu_{t}\right)}\left(S-S_{0}\right)^{2}-\left(\mu_{a}+\eta+\mu_{t}\right) A T \\
& +\frac{\left(\sigma_{1} S\right)^{2}+\left(\sigma_{2} A\right)^{2}+\left(\sigma_{3} T\right)^{2}}{2}=\left[-\mu_{s}+\frac{\left(\mu_{s}+\mu_{a}\right)^{2}}{2 \mu_{a}}\right. \\
& \left.+\frac{\left(\mu_{s}+\eta+\mu_{t}\right)^{2}}{2\left(\eta+\mu_{t}\right)}\right]\left(S-S_{0}\right)^{2}-\frac{\mu_{a}}{2} A^{2}-\frac{\eta+\mu_{t}}{2} T^{2} \\
& -\left(\mu_{a}+\eta+\mu_{t}\right) A T+\frac{\left(\sigma_{1} S\right)^{2}+\left(\sigma_{2} A\right)^{2}+\left(\sigma_{3} T\right)^{2}}{2} .
\end{aligned}
$$

In order to eliminate the cross term $A T$, we consider linear combination of $V_{3}$ and $V_{4}$ as $\left(\left(\mu_{a}+\eta+\mu_{t}\right) / \xi_{1}\right) V_{3}+V_{4}$; then

$$
\begin{aligned}
L[ & \left.\frac{\mu_{a}+\eta+\mu_{t}}{\xi_{1}} V_{3}+V_{4}\right] \\
\leq & {\left[-\mu_{s}+\frac{\left(\mu_{s}+\mu_{a}\right)^{2}}{2 \mu_{a}}+\frac{\left(\eta+\mu_{t}+\mu_{s}\right)^{2}}{2\left(\eta+\mu_{t}\right)}\right]\left(S-S_{0}\right)^{2} } \\
& \quad-\frac{\mu_{a}}{2} A^{2}-\frac{\eta+\mu_{t}}{2} T^{2} \\
& +\frac{\left(\sigma_{1} S\right)^{2}+\left(\sigma_{2} A\right)^{2}+\left(\sigma_{3} T\right)^{2}}{2} \\
& +\frac{\mu_{a}+\eta+\mu_{t}}{\xi_{1}} \frac{\left(\sigma_{3} T\right)^{2}}{2} .
\end{aligned}
$$

At last, to eliminate the term $\left(S-S_{0}\right)^{2}$, we consider linear combination

$$
\begin{aligned}
\frac{1}{\mu_{s}} & {\left[\frac{\left(\mu_{s}+\mu_{a}\right)^{2}}{2 \mu_{a}}+\frac{\left(\eta+\mu_{t}+\mu_{s}\right)^{2}}{2\left(\eta+\mu_{t}\right)}\right]\left(V_{1}+S_{0} V_{2}\right) } \\
& +\frac{\mu_{a}+\eta+\mu_{t}}{\xi_{1}} V_{3}+V_{4} .
\end{aligned}
$$

Let $V=\left(1 / \mu_{s}\right)\left[\left(\mu_{s}+\mu_{a}\right)^{2} / 2 \mu_{a}+\left(\mu_{s}+\eta+\mu_{t}\right)^{2} / 2\left(\eta+\mu_{t}\right)\right]\left(V_{1}+\right.$ $\left.S_{0} V_{2}\right)+\left(\left(\mu_{a}+\eta+\mu_{t}\right) / \xi_{1}\right) V_{3}+V_{4}$. Integrating all the formulas of $L V_{i}$, then

$$
\begin{aligned}
L V & \leq-\mu_{s}\left(S-S_{0}\right)^{2}-\frac{\mu_{a}}{2} A^{2}-\frac{\eta+\mu_{t}}{2} T^{2} \\
& +\left[\frac{1}{2 \mu_{s}}\left(\frac{\left(\mu_{s}+\mu_{a}\right)^{2}}{2 \mu_{a}}+\frac{\left(\mu_{s}+\eta+\mu_{t}\right)^{2}}{2\left(\eta+\mu_{t}\right)}\right)+\frac{1}{2}\right]
\end{aligned}
$$

$$
\begin{aligned}
& \cdot\left(\sigma_{1} S\right)^{2}+\frac{1}{2}\left(\sigma_{2} A\right)^{2}+\left(\frac{1}{2}+\frac{\mu_{a}+\eta+\mu_{t}}{2 \xi_{1}}\right)\left(\sigma_{3} T\right)^{2} \\
& :=-\mu_{s}\left(S-S_{0}\right)^{2}-\frac{\mu_{a}}{2} A^{2}-\frac{\eta+\mu_{t}}{2} T^{2}+c_{1} S^{2}+c_{2} A^{2} \\
& +c_{3} T^{2}
\end{aligned}
$$

where $c_{i}(i=1,2,3)$ is defined as follows:

$$
\begin{aligned}
& c_{1}=\left[\frac{1}{2 \mu_{s}}\left(\frac{\left(\mu_{s}+\mu_{a}\right)^{2}}{2 \mu_{a}}+\frac{\left(\mu_{s}+\eta+\mu_{t}\right)^{2}}{2\left(\eta+\mu_{t}\right)}\right)+\frac{1}{2}\right] \sigma_{1}^{2}, \\
& c_{2}=\frac{\sigma_{2}^{2}}{2} \\
& c_{3}=\left(\frac{1}{2}+\frac{\mu_{a}+\eta+\mu_{t}}{2 \xi_{1}}\right) \sigma_{3}^{2} .
\end{aligned}
$$

Seen from the definition of $V_{i}, i=1,2,3,4$,

$$
\begin{aligned}
V= & \frac{1}{\mu_{s}}\left[\frac{\mu_{s}+\mu_{a}}{2 \mu_{a}}+\frac{\eta+\mu_{t}+\mu_{s}}{2\left(\eta+\mu_{t}\right)}\right]\left(V_{1}+S_{0} V_{2}\right) \\
& +\frac{\eta+\mu_{a}+\mu_{t}}{\xi_{1}} V_{3}+V_{4}
\end{aligned}
$$

is obviously positive definite; it is easy to know that

$$
\begin{aligned}
\lim _{T \rightarrow \infty} & \frac{1}{T} \int_{0}^{T}\left[\left(\mu_{s}-c_{1}\right)\left(S(t)-S_{0}\right)^{2}\right. \\
& +\left(\frac{\mu_{a}}{2} A^{2}-c_{2}\right) A^{2}(t) \\
& \left.+\left(\frac{\eta+\mu_{t}}{2}-c_{3}\right) T^{2}(t)\right] d t \leq c_{1} S_{0}^{2} .
\end{aligned}
$$

The proof is complete.

Remark 7. Besides, $Q_{0}$ becomes the disease-free equilibrium of system (1) as $\sigma_{1}=0$. In the proof, we see

$$
\begin{aligned}
L V \leq & -\mu_{s}\left(S-S_{0}\right)^{2}-\left(\frac{\mu_{a}}{2}-c_{2}\right) A^{2} \\
& -\left(\frac{\eta+\mu_{t}}{2}-c_{3}\right) T^{2},
\end{aligned}
$$

Thus, the solution of system is stochastically asymptotically stable in the large [22] as the conditions $\mu_{a} / 2>c_{2}$, $\left(\eta+\mu_{t}\right) / 2>c_{3}$ are satisfied.

\section{Stochastic Stability of the Internal Equilibrium Point $E^{*}$}

In this section, we will investigate the stochastic behavior of (5) around the internal equilibrium point $E^{*}$ in deterministic model (13) from two aspects; that is, one is concerned with the stability of its distribution, the other is concerned with ergodic property of equilibrium point $E^{*}$. 
Theorem 8. If the following conditions are satisfied,

$$
\begin{aligned}
\mu_{s} & >\frac{C}{S^{* 2}}+c_{1}, \\
\mu_{a} & >2\left(\frac{C}{A^{* 2}}+c_{2}\right), \\
\eta+\mu_{t} & >2\left(\frac{C}{T^{* 2}}+c_{3}\right),
\end{aligned}
$$

where

$$
\begin{aligned}
c_{1}= & \sigma_{1}^{2}+\left[\frac{\left(\mu_{s}+\mu_{a}\right)^{2}}{2 \mu_{a}}+\frac{\left(\mu_{s}+\mu_{t}\right)^{2}}{2\left(\eta+\mu_{t}\right)}\right] \frac{\sigma_{1}^{2}}{\mu_{s}}, \\
c_{2}= & \sigma_{2}^{2}, \\
c_{3}= & \sigma_{3}^{2}+\frac{\mu_{a}+\mu_{t}}{\xi_{1}} \sigma_{1}^{2}, \\
c_{4}= & \frac{1}{\mu_{s}}\left[\frac{\left(\mu_{s}+\mu_{a}\right)^{2}}{2 \mu_{a}}+\frac{\left(\mu_{s}+\mu_{t}\right)^{2}}{2\left(\eta+\mu_{t}\right)}\right] \\
& \cdot\left(\frac{S^{*}}{1+\gamma A^{*}} \frac{\sigma_{2}^{2}}{2}+\frac{\xi_{2} T^{* 2}}{\xi_{1} A^{*}} \frac{S^{*}}{1+\gamma A^{*}} \frac{\sigma_{3}^{2}}{2}\right), \\
C= & c_{1} S^{* 2}+c_{2} A^{* 2}+c_{3} T^{* 2}+c_{4},
\end{aligned}
$$

then (5) is ergodic and positive recurrent.

Proof. Construct Lyapunov function $U_{1}$ as

$$
U_{1}=\frac{\left(S-S^{*}\right)^{2}}{2}
$$

and calculate to get

$$
\begin{aligned}
& L U_{1}=\left(S-S^{*}\right)\left(\Pi-\frac{\beta S A}{1+\gamma A}-\mu_{S} S\right)+\frac{\sigma_{1}^{2}}{2} S^{2} \\
& =\left(S-S^{*}\right)\left[\frac{\beta S^{*} A^{*}}{1+\gamma A^{*}}-\frac{\beta S A}{1+\gamma A}-\mu_{s}\left(S-S^{*}\right)\right] \\
& +\frac{\sigma_{1}^{2}}{2} S^{2}=\beta\left(S-S^{*}\right)\left(\frac{S^{*} A^{*}}{1+\gamma A^{*}}-\frac{S A}{1+\gamma A}\right) \\
& -\mu_{s}\left(S-S^{*}\right)^{2}+\frac{\sigma_{1}^{2}}{2} S^{2}=\beta\left(S-S^{*}\right) \\
& \cdot\left[S^{*} A^{*}\left(\frac{1}{1+\gamma A^{*}}-\frac{1}{1+\gamma A}\right)+\frac{S^{*} A^{*}-S A}{1+\gamma A}\right] \\
& -\mu_{s}\left(S-S^{*}\right)^{2}+\frac{\sigma_{1}^{2}}{2} S^{2}=\beta \gamma S^{*} A^{*} \\
& \frac{\left(A-A^{*}\right)\left(S-S^{*}\right)}{\left(1+\gamma A^{*}\right)(1+\gamma A)}-\frac{\beta S^{*}\left(S-S^{*}\right)\left(A-A^{*}\right)}{1+\gamma A}
\end{aligned}
$$

$$
\begin{aligned}
& -\frac{\beta A\left(S-S^{*}\right)^{2}}{1+\gamma A}-\mu_{s}\left(S-S^{*}\right)^{2}+\frac{\sigma_{1}^{2}}{2} S^{2} \leq-\beta S^{*} \\
& \cdot \frac{\left(S-S^{*}\right)\left(A-A^{*}\right)}{(1+\gamma A)\left(1+\gamma A^{*}\right)}+\frac{\left(\sigma_{1} S\right)^{2}}{2}-\mu_{s}\left(S-S^{*}\right)^{2} .
\end{aligned}
$$

Next, we let

$$
U_{2}=A-A^{*}-A^{*} \ln \frac{A}{A^{*}},
$$

and calculate to get

$$
\begin{aligned}
& L U_{2}=\left(1-\frac{A^{*}}{A}\right)\left(\frac{\beta S A}{1+\gamma A}-\xi_{1} A+\xi_{2} T-\mu_{a} A\right) \\
& +\frac{\sigma_{2}^{2}}{2} \\
& =\left(1-\frac{A^{*}}{A}\right)\left[\frac{\beta S A}{1+\gamma A}-\left(\xi_{1}+\mu_{a}\right) A\right] \\
& +\left(1-\frac{A^{*}}{A}\right) \xi_{2} T+\frac{\sigma_{2}^{2}}{2} \\
& =\left(A-A^{*}\right)\left[\frac{\beta S}{1+\gamma A}-\left(\xi_{1}+\mu_{a}\right)\right]+\xi_{2} T \\
& -\xi_{2} T \frac{A^{*}}{A}+\frac{\sigma_{2}^{2}}{2} \\
& =\left(A-A^{*}\right)\left[\frac{\beta S}{1+\gamma A}-\left(\frac{\beta S^{*}}{1+\gamma A^{*}}+\xi_{2} \frac{T^{*}}{A^{*}}\right)\right] \\
& +\xi_{2} T-\xi_{2} T \frac{A^{*}}{A}+\frac{\sigma_{2}^{2}}{2} \\
& =\beta\left(A-A^{*}\right)\left(\frac{S}{1+\gamma A}-\frac{S^{*}}{1+\gamma A^{*}}\right) \\
& +\frac{\xi_{2} T^{*}}{A^{*}}\left(A-A^{*}\right)+\xi_{2} T-\xi_{2} T \frac{A^{*}}{A}+\frac{\sigma_{2}^{2}}{2} \\
& =\frac{\beta\left(A-A^{*}\right)\left(S-S^{*}\right)}{1+\gamma A} \\
& +\beta S^{*}\left(A-A^{*}\right)\left(\frac{1}{1+\gamma A}-\frac{1}{1+\gamma A^{*}}\right) \\
& +\frac{\xi_{2} T^{*}}{A^{*}}\left(A-A^{*}\right)+\xi_{2} T-\xi_{2} T \frac{A^{*}}{A}+\frac{\sigma_{2}^{2}}{2} \\
& \leq \frac{\beta\left(A-A^{*}\right)\left(S-S^{*}\right)}{1+\gamma A}+\frac{\xi_{2} T^{*}}{A^{*}}\left(A-A^{*}\right)+\xi_{2} T \\
& -\xi_{2} T \frac{A^{*}}{A}+\frac{\sigma_{2}^{2}}{2} \text {. }
\end{aligned}
$$


Then, it is easy to know

$$
\begin{aligned}
& L\left(U_{1}+\frac{S^{*}}{1+\gamma A^{*}} U_{2}\right) \leq-\mu_{s}\left(S-S^{*}\right)^{2}+\frac{\sigma_{1}^{2} S^{2}}{2} \\
& +\frac{S^{*}}{1+\gamma A^{*}} \frac{\xi_{2} T^{*}}{A^{*}}\left(A-A^{*}\right)+\frac{S^{*}}{1+\gamma A^{*}} \xi_{2} T \\
& \quad-\frac{S^{*}}{1+\gamma A^{*}} \xi_{2} T \frac{A^{*}}{A}+\frac{S^{*}}{1+\gamma A^{*}} \frac{\sigma_{2}^{2}}{2}=-\mu_{s}\left(S-S^{*}\right)^{2} \\
& +\frac{\sigma_{1}^{2} S^{2}}{2}+\frac{S^{*}}{1+\gamma A^{*}} \frac{\xi_{2} T^{*}}{A^{*}} A+\frac{S^{*}}{1+\gamma A^{*}} \xi_{2}\left(T-T^{*}\right) \\
& +\frac{S^{*}}{1+\gamma A^{*}} \xi_{2} T \frac{A^{*}}{A}+\frac{S^{*}}{1+\gamma A^{*}} \frac{\sigma_{2}^{2}}{2}=-\mu_{s}\left(S-S^{*}\right)^{2} \\
& +\frac{\sigma_{1}^{2} S^{2}}{2}-\frac{S^{*}}{1+\gamma A^{*}} \\
& +\frac{\sigma_{1}^{2} S^{2}}{2}+\frac{S^{*}}{1+\gamma A^{*}} \frac{\sigma_{2}^{2}}{2}, \quad \xi_{2} T^{*}\left[\left(\frac{A}{A^{*}}+\frac{T}{T^{*}}+\frac{T}{T^{*}} \frac{A^{*}}{A}-1\right)\right]+\frac{\sigma_{1}^{2} S^{2}}{2} \\
& +\frac{S^{*}}{1+\gamma A^{*}} \frac{\sigma_{2}^{2}}{2} \leq-\mu_{s}\left(S-S^{*}\right)^{2}-\frac{S^{*}}{1+\gamma A^{*}} \\
& +\xi_{2} T^{*}\left(\frac{A}{A^{*}}-\log \frac{A^{*}}{A}-1+\frac{T^{*}}{T}-1\right)
\end{aligned}
$$

where the last inequality is derived from $x \geq \log x+1, x>0$.

Let

$$
U_{3}=\frac{\left(S-S^{*}+A-A^{*}+T-T^{*}\right)^{2}}{2} ;
$$

we calculate to get

$$
\begin{aligned}
& L U_{3}=\left(S-S^{*}+A-A^{*}+T-T^{*}\right)\left[\Pi-\mu_{s} S-\mu_{a} A\right. \\
& \left.-\left(\eta+\mu_{t}\right) T\right]+\frac{\sigma_{1}^{2}}{2} S^{2}+\frac{\sigma_{2}^{2}}{2} A^{2}+\frac{\sigma_{3}^{2}}{2} T^{2}=\left(S-S^{*}\right. \\
& \left.+A-A^{*}+T-T^{*}\right)\left[-\mu_{s}\left(S-S^{*}\right)-\mu_{a}\left(A-A^{*}\right)\right. \\
& \left.-\left(\eta+\mu_{t}\right)\left(T-T^{*}\right)\right]+\frac{\sigma_{1}^{2}}{2} S^{2}+\frac{\sigma_{2}^{2}}{2} A^{2}+\frac{\sigma_{3}^{2}}{2} T^{2} \\
& =-\mu_{s}\left(S-S^{*}\right)^{2}-\mu_{a}\left(A-A^{*}\right)^{2}-\left(\eta+\mu_{t}\right)(T \\
& \left.-T^{*}\right)^{2}-\left(\mu_{s}+\mu_{a}\right)\left(S-S^{*}\right)\left(A-A^{*}\right)-\left(\mu_{s}+\mu_{t}\right) \\
& \cdot\left(S-S^{*}\right)\left(T-T^{*}\right)-\left(\mu_{a}+\mu_{t}\right)\left(A-A^{*}\right)\left(T-T^{*}\right) \\
& +\frac{\sigma_{1}^{2}}{2} S^{2}+\frac{\sigma_{2}^{2}}{2} A^{2}+\frac{\sigma_{3}^{2}}{2} T^{2} .
\end{aligned}
$$

Let

$$
U_{4}=\frac{\left(T-T^{*}\right)^{2}}{2}
$$

similar to the technique in computing $L U_{1}$, we calculate to get $\mathrm{LU}_{4}$

$$
\begin{aligned}
= & \left(T-T^{*}\right)\left[\xi_{1}\left(A-A^{*}\right)-\left(\xi_{2}+\eta+\mu_{t}\right)\left(T-T^{*}\right)\right] \\
& +\frac{\sigma_{1}^{2}}{2} T^{2} \\
= & \xi_{1}\left(A-A^{*}\right)\left(T-T^{*}\right)-\left(\xi_{2}+\eta+\mu_{t}\right)\left(T-T^{*}\right)^{2} \\
& +\frac{\sigma_{1}^{2}}{2} T^{2} .
\end{aligned}
$$

Considering the linear combination of $U_{3}$ and $U_{4}$ as

$$
U_{3}+\frac{\mu_{a}+\mu_{t}}{\xi_{1}} U_{4}
$$

then

$$
\begin{aligned}
L\left[U_{3}+\frac{\mu_{a}+\mu_{t}}{\xi_{1}} U_{4}\right] & \\
= & -\mu_{s}\left(S-S^{*}\right)^{2}-\frac{\mu_{a}}{2}\left(A-A^{*}\right)^{2} \\
& -\left(\frac{\eta+\mu_{t}}{2}+\frac{\mu_{a}+\mu_{t}}{\xi_{1}}\left(\xi_{2}+\eta+\mu_{t}\right)\right)\left(T-T^{*}\right)^{2} \\
& +\frac{\left(\mu_{s}+\mu_{a}\right)^{2}}{2 \mu_{a}}\left(S-S^{*}\right)^{2}+\frac{\left(\mu_{s}+\mu_{t}\right)^{2}}{2\left(\eta+\mu_{t}\right)}\left(S-S^{*}\right)^{2} \\
& +\frac{\mu_{a}+\mu_{t}}{\xi_{1}} \frac{\sigma_{1}^{2}}{2} T^{2}+\frac{\sigma_{1}^{2}}{2} S^{2}+\frac{\sigma_{2}^{2}}{2} A^{2}+\frac{\sigma_{3}^{2}}{2} T^{2} .
\end{aligned}
$$

Next, we define

$$
U_{5}=T-T^{*}-T^{*} \log \frac{T}{T^{*}}
$$

then $\xi_{1} A^{*}=\left(\xi_{2}+\eta+\mu_{t}\right) T^{*}$ implies

$$
\begin{aligned}
L U_{5} & =\left(1-\frac{T^{*}}{T}\right)\left(\xi_{1} A-\left(\xi_{2}+\eta+\mu_{t}\right) T\right)+\frac{\sigma_{3}^{2} T^{*}}{2} \\
= & \xi_{1} A^{*}\left(\frac{A}{A^{*}}-\frac{A}{A^{*}} \frac{T^{*}}{T}-\frac{T}{T^{*}}+1\right)+\frac{\sigma_{3}^{2} T^{*}}{2} \\
\leq & \xi_{1} A^{*}\left[\frac{A}{A^{*}}-\log \frac{A}{A^{*}}-1-\left(\frac{T}{T^{*}}-\log \frac{T}{T^{*}}-1\right)\right] \\
& +\frac{\sigma_{3}^{2} T^{*}}{2} .
\end{aligned}
$$

We let

$$
V=U_{1}+\frac{S^{*}}{1+\gamma A^{*}} U_{2}+\frac{\xi_{2} T^{*}}{\xi_{1} A^{*}} \frac{S^{*}}{1+\gamma A^{*}} U_{5}
$$

it is easy to prove that

$$
\begin{aligned}
L V \leq & -\mu_{s}\left(S-S^{*}\right)^{2}+\frac{\sigma_{1}^{2} S^{2}}{2}+\frac{S^{*}}{1+\gamma A^{*}} \frac{\sigma_{2}^{2}}{2} \\
& +\frac{\xi_{2} T^{* 2}}{\xi_{1} A^{*}} \frac{S^{*}}{1+\gamma A^{*}} \frac{\sigma_{3}^{2}}{2} .
\end{aligned}
$$


We consider the linear combination of

$$
\left[\frac{\left(\mu_{s}+\mu_{a}\right)^{2}}{2 \mu_{a}}+\frac{\left(\mu_{s}+\mu_{t}\right)^{2}}{2\left(\eta+\mu_{t}\right)}\right] \frac{V}{\mu_{s}}+U_{3}+\frac{\mu_{a}+\mu_{t}}{\xi_{1}} U_{4}
$$

then

$$
\begin{aligned}
& L\left[\left(\frac{\left(\mu_{s}+\mu_{a}\right)^{2}}{2 \mu_{a}}+\frac{\left(\mu_{s}+\mu_{t}\right)^{2}}{2\left(\eta+\mu_{t}\right)}\right) \frac{V}{\mu_{s}}+U_{3}\right. \\
& \left.\quad+\frac{\mu_{a}+\mu_{t}}{\xi_{1}} U_{4}\right] \leq-\mu_{s}\left(S-S^{*}\right)^{2}-\frac{\mu_{a}}{2}\left(A-A^{*}\right)^{2} \\
& \quad-\left(\frac{\eta+\mu_{t}}{2}+\frac{\mu_{a}+\mu_{t}}{\xi_{1}}\left(\xi_{2}+\eta+\mu_{t}\right)\right)\left(T-T^{*}\right)^{2} \\
& +\frac{c_{1} S^{2}}{2}+\frac{c_{2} A^{2}}{2}+\frac{c_{3} T^{2}}{2}+c_{4} \leq-\left(\mu_{s}-c_{1}\right)\left(S-S^{*}\right)^{2} \\
& \quad-\left(\frac{\mu_{a}}{2}-c_{2}\right)\left(A-A^{*}\right)^{2}-\left(\frac{\eta+\mu_{t}}{2}-c_{3}\right)\left(T-T^{*}\right)^{2} \\
& +C
\end{aligned}
$$

where $c_{i}, i=1,2,3,4$ is represented as

$$
\begin{aligned}
c_{1}= & \sigma_{1}^{2}+\left[\frac{\left(\mu_{s}+\mu_{a}\right)^{2}}{2 \mu_{a}}+\frac{\left(\mu_{s}+\mu_{t}\right)^{2}}{2\left(\eta+\mu_{t}\right)}\right] \frac{\sigma_{1}^{2}}{\mu_{s}}, \\
c_{2}= & \sigma_{2}^{2}, \\
c_{3}= & \sigma_{3}^{2}+\frac{\mu_{a}+\mu_{t}}{\xi_{1}} \sigma_{1}^{2}, \\
c_{4}= & \frac{1}{\mu_{s}}\left[\frac{\left(\mu_{s}+\mu_{a}\right)^{2}}{2 \mu_{a}}+\frac{\left(\mu_{s}+\mu_{t}\right)^{2}}{2\left(\eta+\mu_{t}\right)}\right] \\
& \cdot\left(\frac{S^{*}}{1+\gamma A^{*}} \frac{\sigma_{2}^{2}}{2}+\frac{\xi_{2} T^{* 2}}{\xi_{1} A^{*}} \frac{S^{*}}{1+\gamma A^{*}} \frac{\sigma_{3}^{2}}{2}\right), \\
C= & c_{4}+c_{1}\left(S^{*}\right)^{2}+c_{2}\left(A^{*}\right)^{2}+c_{3}\left(T^{*}\right)^{2}
\end{aligned}
$$

Choose appropriate parameters in system (2) to satisfy

$$
\begin{array}{r}
\left(\mu_{s}-c_{1}\right)\left(S^{*}\right)^{2}>C, \\
\left(\frac{\mu_{a}}{2}-c_{2}\right)\left(A^{*}\right)^{2}>C, \\
\left(\frac{\eta+\mu_{t}}{2}-c_{3}\right)\left(T^{*}\right)^{2}>C ;
\end{array}
$$

that is,

$$
\begin{aligned}
\mu_{s} & >\frac{C}{\left(S^{*}\right)^{2}}+c_{1}, \\
\mu_{a} & >2\left(\frac{C}{\left(A^{*}\right)^{2}}+c_{2}\right), \\
\eta+\mu_{t} & >2\left(\frac{C}{\left(T^{*}\right)^{2}}+c_{3}\right) .
\end{aligned}
$$

Hence, if we set

$$
U=\left[\frac{\left(\mu_{s}+\mu_{a}\right)^{2}}{2 \mu_{a}}+\frac{\left(\mu_{s}+\mu_{t}\right)^{2}}{2\left(\eta+\mu_{t}\right)}\right] \frac{V}{\mu_{s}}+U_{3}+\frac{\mu_{a}+\mu_{t}}{\xi_{1}} U_{4}
$$

$>0$,

then $L U \leq-C$ for some $C>0$. The proof is complete.

Next, we will discuss the mean convergence of system (5) based on the condition in Theorem 6 .

Theorem 9. If the condition in Theorem 6 holds, then the following conclusions are established:

$$
\begin{aligned}
& \lim _{T \rightarrow \infty} \frac{1}{T} \int_{0}^{T} \frac{\beta S(u) A(u)}{1+\gamma A(u)} d u+\lim _{T \rightarrow \infty} \frac{1}{T} \int_{0}^{T} \mu_{s} S(u) d u \\
& \quad=\Pi, \text { a.e., } \\
& \lim _{T \rightarrow \infty} \frac{1}{T} \int_{0}^{T} \frac{\beta S(u) A(u)}{1+\gamma A(u)} d u+\lim _{T \rightarrow \infty} \frac{1}{T} \int_{0}^{T} \xi_{2} T(u) d u \\
& \quad=\lim _{T \rightarrow \infty} \frac{1}{T} \int_{0}^{T}\left[\xi_{1} A(u)+\mu_{a} A(u)\right] d u, \quad \text { a.e, } \\
& \lim _{T \rightarrow \infty} \frac{1}{T} \int_{0}^{T} \xi_{1} A(u) d u \\
& =\lim _{T \rightarrow \infty} \frac{1}{T} \int_{0}^{T}\left[\xi_{2} T(u)+\eta T(u)+\mu_{t} T(u)\right] d u,
\end{aligned}
$$

a.e.

Proof. By ergodic theorem, for arbitrary function $f$, if it satisfies

$$
\int|f(x, y, z) m(d x, d y, d z)|<+\infty
$$

then

$$
\begin{aligned}
\lim _{T \rightarrow \infty} \frac{1}{T} \int_{0}^{T} f(S(u), A(u), T(u)) d u & \longrightarrow \\
& \quad \int f(x, y, z) m(d x, d y, d z)<+\infty, \quad \text { a.e. }
\end{aligned}
$$

Note $C=\min \left\{\mu_{s}, \mu_{a}, \eta+\mu_{t}\right\}$, since

$$
\begin{aligned}
d(S(t) & +A(t)+T(t)) \\
= & \Pi-\mu_{s} S(t)-\mu_{a} A(t)-\left(\eta+\mu_{t}\right) T(t) \\
& +\sigma_{1} S(t) d B_{1}(t)+\sigma_{2} A(t) d B_{2}(t) \\
& +\sigma_{3} T(t) d B_{3}(t) ;
\end{aligned}
$$


we take stochastic integral of the above formula over $[0, t]$, $0<t<T$,

$$
\begin{aligned}
& S(t)+ A(t)+T(t) \\
&= S(0)+A(0)+T(0)+\Pi t \\
&-\int_{0}^{t}\left[\mu_{s} S(u)+\mu_{a} A(u)+\left(\eta+\mu_{t}\right) T(u)\right] d u \\
&+\int_{0}^{t} \sigma_{1} S(u) d B_{1}(u)+\int_{0}^{t} \sigma_{2} A(u) d B_{2}(u) \\
&+\int_{0}^{t} \sigma_{3} T(u) d B_{3}(u), \\
& E[S(t)+A(t)+T(t)] \\
& \leq S(0)+A(0)+T(0) \\
& \quad-C \int_{0}^{t} E[S(u)+A(u)+T(u)] d u
\end{aligned}
$$

by Gronwall inequality, we can derive

$$
0 \leq E[S(t)+A(t)+T(t)]<M<+\infty .
$$

Furthermore,

$$
\begin{array}{r}
\lim _{T \rightarrow \infty} \frac{1}{T} \int_{0}^{T}[S(t)+A(t)+T(t)] \wedge n d t \\
\quad=\int(x+y+z) \wedge n m(d x, d y, d z) .
\end{array}
$$

After taking expectation of this formula, by the control convergence theorem, we know

$$
\int_{R_{+}^{3}}(x+y+z) \wedge n m(d x, d y, d z) \leq M<+\infty .
$$

Let $n \rightarrow+\infty$; then

$$
\int_{R_{+}^{3}}(x+y+z) m(d x, d y, d z)<+\infty .
$$

Therefore, if we define $f=\beta S A /(1+\gamma A)$ or $S$ or $A$ or $T$, respectively, we can prove the corresponding convergence.

By the use of (5), we can get

$$
\begin{aligned}
& S(t)=\int_{0}^{t}\left[\left(\Pi-\frac{\beta S(u) A(u)}{1+\gamma A(u)}-\mu_{s} S(u)\right) d u\right. \\
& \left.+\sigma_{1} S(u) d B_{1}(u)\right] .
\end{aligned}
$$

Hence,

$$
\begin{aligned}
\frac{S(t)}{t}= & \frac{1}{t} \int_{0}^{t}\left[\Pi-\frac{\beta S(u) A(u)}{1+\gamma A(u)}-\mu_{s} S(u)\right] d u \\
& +\frac{1}{t} \int_{0}^{t} \sigma_{1} S(u) d B_{1}(u) .
\end{aligned}
$$

By the law of large numbers of martingale,

$$
\lim _{t \rightarrow \infty} \frac{1}{t} \int_{0}^{t} \sigma_{1} S(u) d B_{1}(u) \longrightarrow 0 .
$$

Considering that $\lim _{t \rightarrow \infty}(S(t) / t)$ exists, then

$$
E \lim _{t \rightarrow \infty} \frac{S(t)}{t}=\lim _{t \rightarrow \infty} E \frac{S(t)}{t} .
$$

Since $E S(t) \leq M, \forall t$, then

$$
E \lim _{t \rightarrow \infty} \frac{S(t)}{t}=0
$$

Thus, we proved

$$
\frac{S(t)}{t} \longrightarrow 0, \quad \text { a.e. }
$$

Similarly, we can prove

$$
\begin{aligned}
& \frac{A(t)}{t} \longrightarrow 0, \\
& \frac{T(t)}{t} \longrightarrow 0,
\end{aligned}
$$

a.e.

From (84), we can conclude

$$
\lim _{T \rightarrow \infty} \frac{1}{T} \int_{0}^{T}\left[\Pi-\frac{\beta S(u) A(u)}{1+\gamma A(u)}-\mu_{s} S(u)\right] d u=0 .
$$

The others conclusions can be similarly proved. The proof is completed.

\section{Numerical Simulations}

In this section, to verify the results of stochastic stability, we use Milstein's numerical method to make corresponding simulations based on stochastic model (5) with some given initial values and parameters [23]. Thus, discreting system (5), we get the following iterative scheme:

$$
\begin{aligned}
S_{i+1}= & S_{i}+\left(\Pi-\frac{\beta S_{i} A_{i}}{1+\gamma A_{i}}-\mu_{s} S_{i}\right) \Delta t \\
& +\sigma_{1} S_{i} c(1, i) \sqrt{\Delta t} \\
& +0.5 \sigma_{1}^{2} S_{i}\left(c(1, i)^{2}-1\right) \Delta t, \\
A_{i+1}= & A_{i}+\left(\frac{\beta S_{i} A_{i}}{1+\gamma A_{i}}-\xi_{1} A_{i}+\xi_{2} T_{i}-\mu_{a} A_{i}\right) \Delta t \\
& +\sigma_{2} A_{i} c(2, i) \sqrt{\Delta t} \\
& +0.5 \sigma_{2}^{2} A_{i}\left(c(2, i)^{2}-1\right) \Delta t, \\
T_{i+1}= & T_{i}+\left(\xi_{1} A_{i}-\xi_{2} T_{i}-\eta T_{i}-\mu_{t} T_{i}\right) \Delta t \\
& +\sigma_{3} T_{i} c(3, i) \sqrt{\Delta t} \\
& +0.5 \sigma_{3}^{2} T_{i}\left(c(2, i)^{2}-1\right) \Delta t,
\end{aligned}
$$




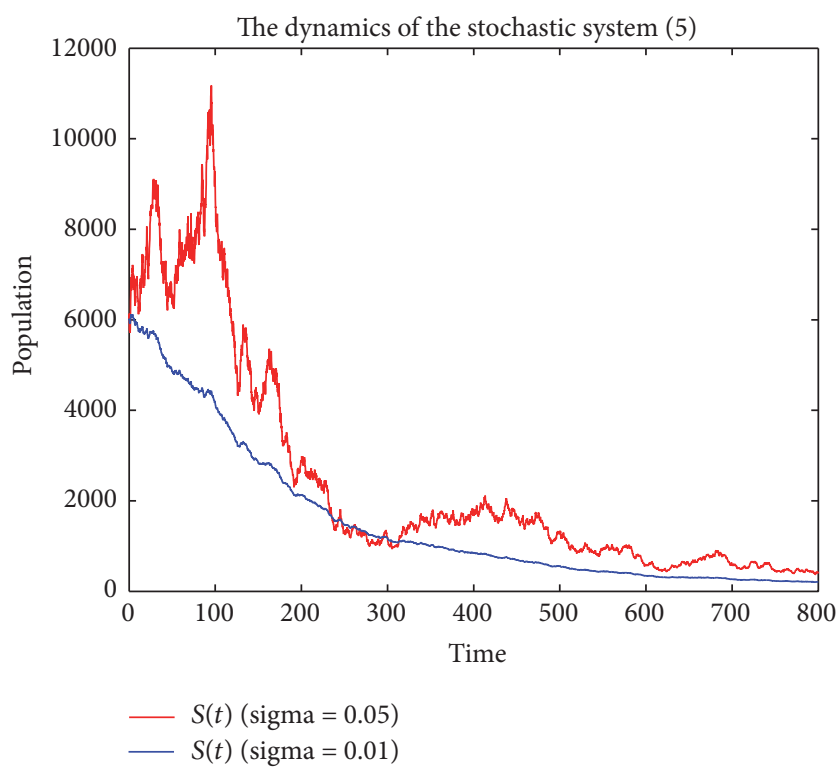

FIGURE 1: $R_{0}<1$, population evolution curve of $S(t)$ with disturbance intensity $\sigma_{i}=0.05,0.01(i=1,2,3,4)$.

$$
\begin{aligned}
Q_{i+1}= & Q_{i}+\left(\eta T_{i}-\mu_{q} Q_{i}\right) \Delta t+\sigma_{4} Q_{i} c(4, i) \sqrt{\Delta t} \\
& +0.5 \sigma_{4}^{2} Q_{i}\left(c(4, i)^{2}-1\right) \Delta t
\end{aligned}
$$

where $c(l, i), l=1,2,3,4 ; i=1,2, \ldots, n$, are independent Gaussian random variables $N(0,1)$ and $\sigma_{i}, i=1,2,3,4$, are intensities of white noise.

We choose appropriate initial values $(S(0), A(0), T(0)$, $Q(0))=(6000,70,20,5)$ and system parameter values as $\gamma=$ 0.2 as well as population mortality are $\mu_{s}=0.001, \mu_{a}=0.005$, $\mu_{t}=0.003$, and $\mu_{q}=0.0015$, respectively, by the latest report from WHO [7]. In addition, some parameters are reasonably estimated as $\eta=0.3, \xi_{1}=\xi_{2}=0.2$. In our simulations, in view of the fact that drinking is related to the people's habit and thus is a long-term behavior, for the sake of simplicity, we will only consider the evolution curves of $S(t)$ and $A(t)$. Furthermore, we will select different critical parameters to discuss stability of the stochastic solution of (5) as follows.

(1) We choose population recruitment rate as $\Pi=0.1$ and alcohol infection rate as $\beta=0.001$ to make $R_{0}=0.797<1$. To compare we let disturbance intensity be $\sigma_{i}=0.05$ and $0.01(i=1,2,3,4$.), respectively (see Figures 1 and 2$)$.

Synthesizing and comparing the information in Figures 1 and 2, we can conclude that when $R_{0}<1$, regardless of the size of the disturbance intensity, the solution of stochastic system (5) will randomly tend to the alcohol-free equilibrium $E_{0}=\left(\Pi / \mu_{s}, 0,0,0\right)$ of deterministic system (13). Specifically speaking, if we can take some effective measures, for example, controlling the population recruitment rate $\Pi$ or reducing the infection rate $\beta$, the population in $A(t), T(t), Q(t)$ will eventually tend to zero while the population in $S(t)$ will eventually tend to a constant $\Pi / \mu_{s}$. Figures 1 and 2 agree with the conclusions in Theorems 6 and 9.

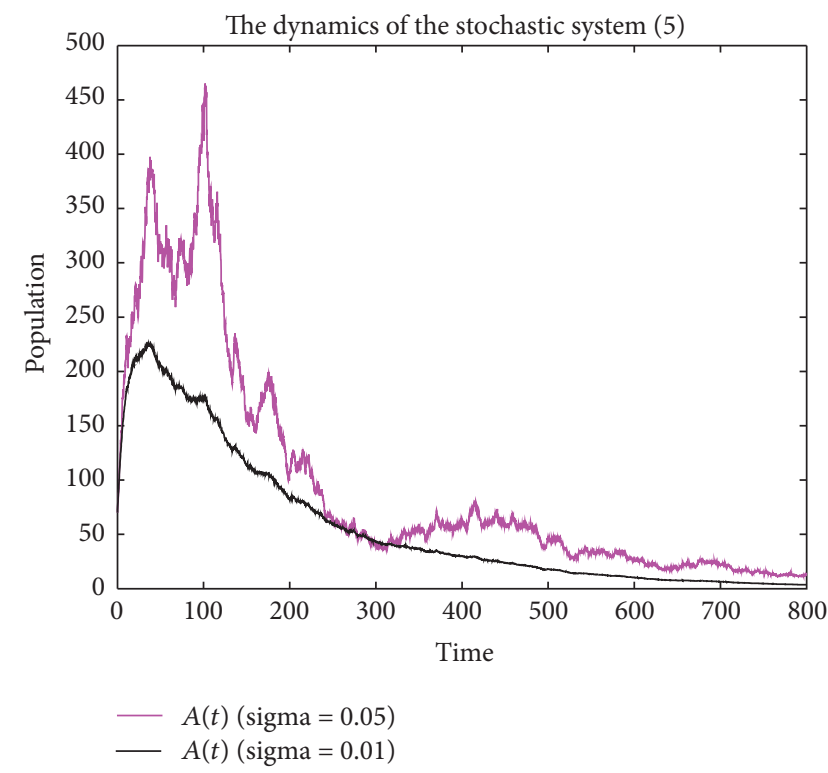

FIgURE 2: $R_{0}<1$, population evolution curve of $A(t)$ with disturbance intensity $\sigma_{i}=0.05,0.01(i=1,2,3,4$. $)$.

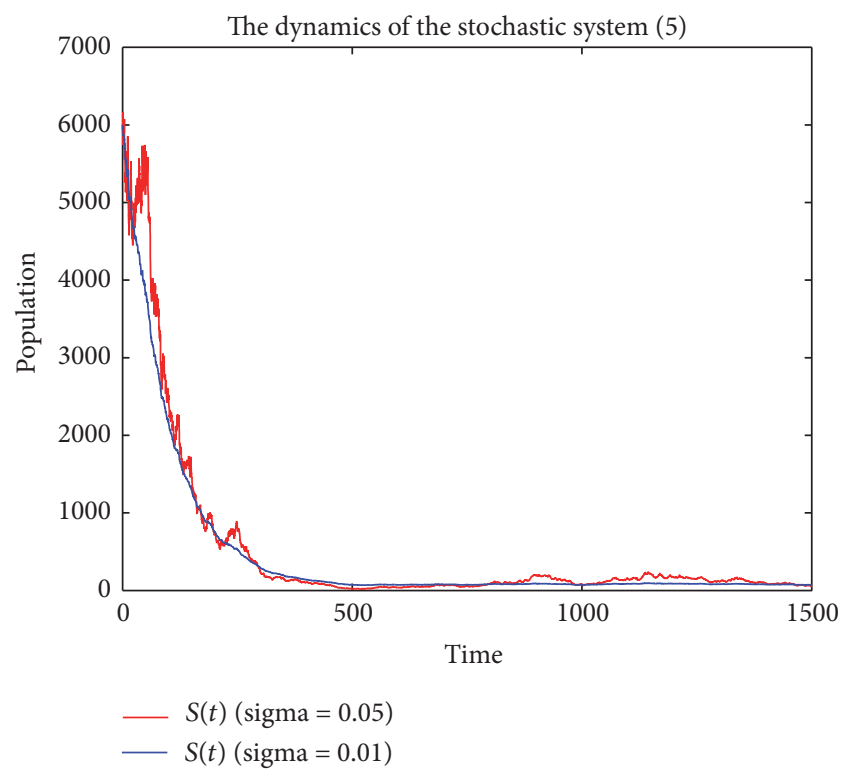

FIgURE 3: $R_{0}>1$, population evolution curve of $S(t)$ with disturbance intensity $\sigma_{i}=0.05,0.01(i=1,2,3,4$.).

(2) We choose population recruitment rate as $\Pi=0.2$ and alcohol infection rate as $\beta=0.002$ to make $R_{0}=1.59>1$. To compare, we still let disturbance intensity be $\sigma_{i}=0.05$ and 0.01 ( $i=1,2,3,4)$, respectively (see Figures 3 and 4 ).

Similarly, by synthesizing and comparing the information in Figures 3 and 4, we can conclude that, in one hand, when $R_{0}>1$, regardless of the size of the disturbance intensity, the solution of stochastic system (5) will randomly tend to the internal equilibrium point $E^{*}=\left(S^{*}, A^{*}, T^{*}, Q^{*}\right)$ of deterministic system corresponding to $\sigma_{i}=0(i=1,2,3,4)$ in 


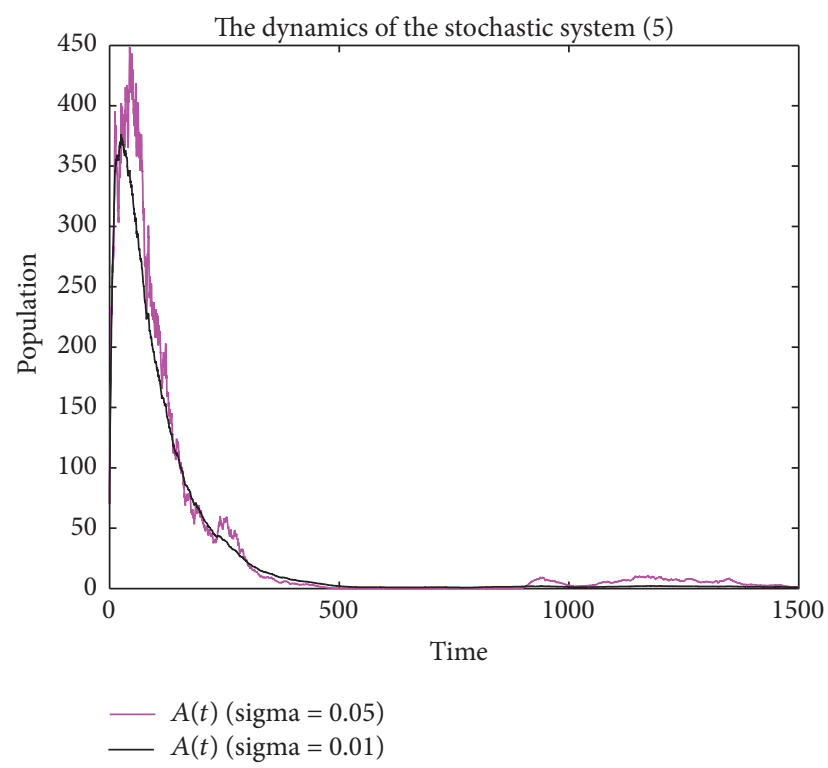

FIGURE 4: $R_{0}>1$, population evolution curve of $A(t)$ with disturbance intensity $\sigma_{i}=0.05,0.01(i=1,2,3,4$. $)$.

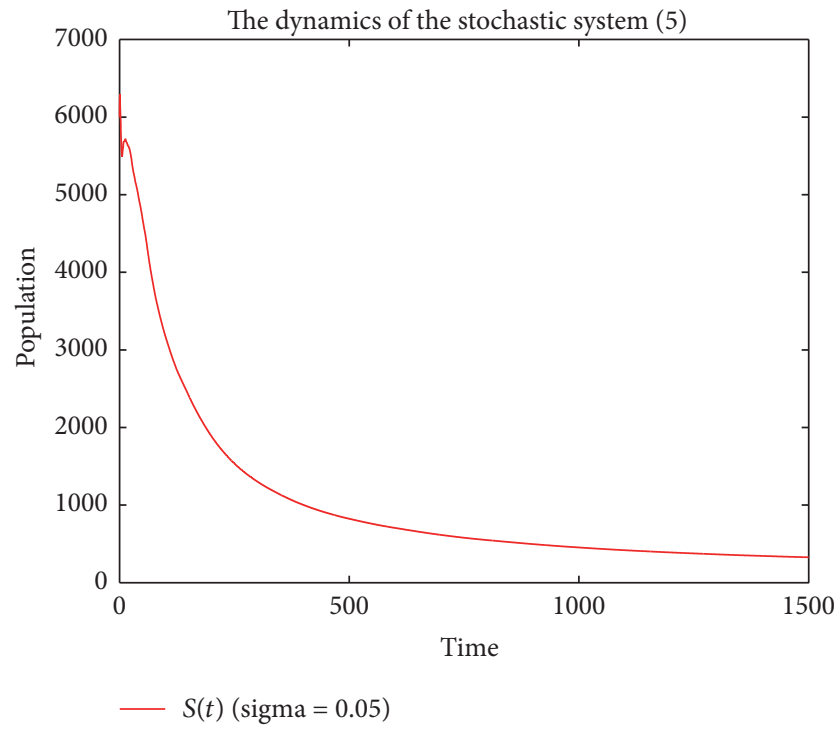

FIGURE 5: $R_{0}>1$, ergodic property of $S(t)$ with disturbance intensity $\sigma_{i}=0.05(i=1,2,3,4$. $)$.

(5). Specifically speaking, if the population recruitment rate $\Pi$ or the infection rate $\beta$ is rather large, the population in $A(t), T(t), Q(t)$ will increase with time going and eventually tends to a fixed level, while the population in $S(t)$ will decrease with time going and eventually tends to a constant. In the other hand, the disturbance intensity of the system will affect the speed of the trend; specifically, the greater the disturbance, the slower this trend, and vice versa. Figures 3 and 4 agree with the conclusions in Theorems 8 and 9.

(3) To testify the ergodic property of system (5), using the same parameters as case (2), we draw Figures 5 and 6 as

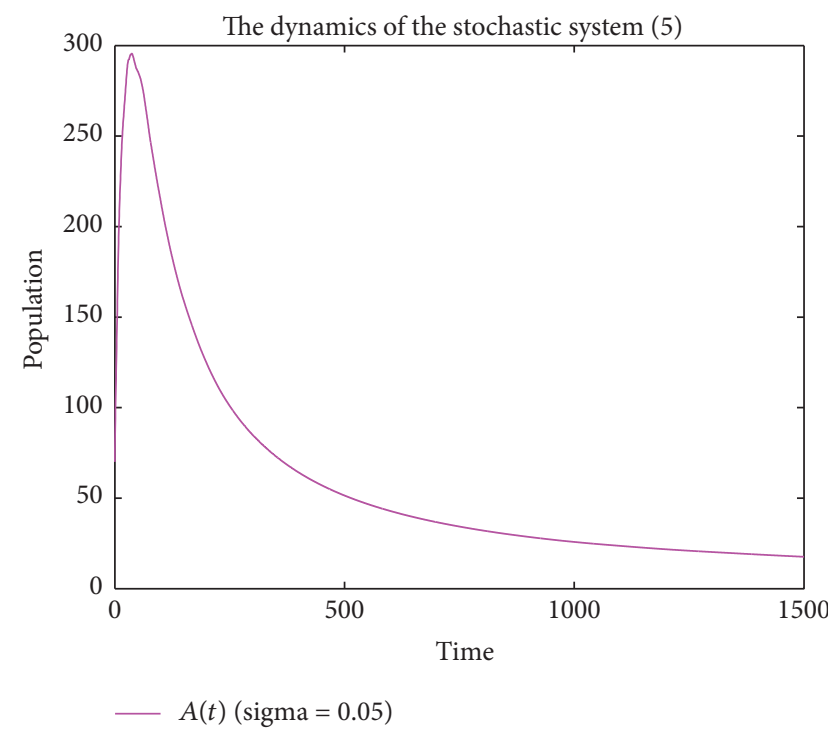

FIGURE 6: $R_{0}>1$, ergodic property of $A(t)$ with disturbance intensity $\sigma_{i}=0.05(i=1,2,3,4$.).

follows only with relatively large disturbance intensity $\sigma_{i}=$ $0.05, i=1,2,3,4$.

Figures 5 and 6 reveal the fact that if system parameters meet the conditions to make $R_{0}>1$, then the model is ergodic and has a uniqueness stationary distribution which illustrates the persistence. This agrees with the conclusions in Theorem 9.

\section{Conclusions}

In this paper, with the fact that the addiction of alcohol abuse complies with the law of infectious diseases being taken into account, we formulate a stochastic alcoholism model to investigate the stochastic stability of the model. We find that, if $R_{0}=\beta\left(\Pi / \mu_{s}\right)\left(\left(\xi_{2}+\eta+\mu_{t}\right) /\left[\xi_{1}\left(\eta+\mu_{t}\right)+\mu_{a}\left(\xi_{2}+\eta+\mu_{t}\right)\right]\right)<1$, the alcohol-free equilibrium $E_{0}=\left(\Pi / \mu_{s}, 0,0,0\right)$ is stochastic asymptotic stability irrelated to system disturbance intensity, which points a way to control alcohol by limiting recruitment to a drinking environment and avoiding contact with the alcoholics as far as possible. On the contrary, when system parameters meet the conditions to make $R_{0}>1$, then the model is ergodic and has a uniqueness stationary distribution which illustrates the persistence. Furthermore, it is worth noting that, in the two cases (i.e., when $R_{0}<1$ or $R_{0}>1$ ), disturbance intensity will obviously affect the trend speed; specifically, the smaller the disturbance, the faster the trend, and vice versa.

\section{Consent}

In this paper, we have quoted some data on population mortality involved with alcoholism, which is published in [7], and these data are authorized by World Health Organization (WHO) to be freely and legally used. 


\section{Competing Interests}

The authors declare that there is no conflict of interests regarding the publication of this paper.

\section{Acknowledgments}

The first author Xun-Yang Wang is supported by the NNSF of China (11461041 and 51369014) and the NSF of Gansu Province (148RJZA024), as well as the Development Program for HongLiu Outstanding Young Teachers in Lanzhou University of Technology. The second author Peng-Zhan Zhang is supported by the NNSF of China (51509122 and 11362010). The corresponding author Qing-Shan Yang is supported by the NNSF of China (11401090).

\section{References}

[1] W. Kornblum and J. Julian, Social Problems, Pearson Education Press, London, UK, 13th edition, 2008.

[2] F. S. Stinson and S. F. Debakey, "Association of alcohol problems with risk for AIDS in the 1988 National Health Interview Survey," Alcohol Health and Research World, vol. 16, no. 3, pp. 245-252, 1992.

[3] C. Carpenter, "Youth alcohol use and risky sexual behavior: evidence from underage drunk driving laws," Journal of Health Economics, vol. 24, no. 3, pp. 613-628, 2005.

[4] N. J. Malunguza, S. D. Hove-Musekwa, G. Musuka, and Z. Mukandavire, "Investigating alcohol consumption as a risk factor for HIV transmission in heterosexual settings in SubSaharan African Communities," Bulletin of Mathematical Biology, vol. 74, no. 9, pp. 2094-2124, 2012.

[5] M. Lyvers, "'Loss of control' in alcoholism and drug addiction: a neuroscientific interpretation," Experimental and Clinical Psychopharmacology, vol. 8, no. 2, pp. 225-249, 2000.

[6] E. E. Epstein, K. Fischer-Elber, and Z. Al-Otaiba, "Women, aging, and alcohol use disorders," Journal of Women and Aging, vol. 19, no. 1-2, pp. 31-48, 2007.

[7] World Health Organization, Global Status Report on Alcohol and Health 2014 Alcohol, World Health Organization, 2014.

[8] F. Sanchez, X. H. Wang, C. C. Chavez et al., "Drinking as an epidemica simple mathematical model with recovery and relapse," in Therapists Guide to Evidence-Based Relapse Prevention: Practical Resources for the Mental Health Professional, K. A. Witkiewitz and G. A. Marlatt, Eds., pp. 353-368, Academic Press, Burlington, Canada, 2007.

[9] G. Mulone and B. Straughan, "Modeling binge drinking," International Journal of Biomathematics, vol. 5, no. 1, Article ID 1250005, pp. 1-14, 2012.

[10] H.-F. Huo and N.-N. Song, "Global stability for a binge drinking model with two stages," Discrete Dynamics in Nature and Society, vol. 2012, Article ID 829386, 15 pages, 2012.

[11] T. K. Greenfield, T. C. Harford, and T. W. Tam, "Modeling cognitive influences on drinking and alcohol problems," Journal of Studies on Alcohol and Drugs, vol. 70, no. 1, pp. 78-86, 2009.

[12] H. Xiang, N.-N. Song, and H.-F. Huo, "Modelling effects of public health educational campaigns on drinking dynamics," Journal of Biological Dynamics, vol. 10, no. 1, pp. 164-178, 2016.

[13] S. Mushayabasa and C. P. Bhunu, "Modelling the effects of heavy alcohol consumption on the transmission dynamics of gonorrhea," Nonlinear Dynamics, vol. 66, no. 4, pp. 695-706, 2011.

[14] S.-H. Ma, H.-F. Huo, and X.-Y. Meng, "Modelling alcoholism as a contagious disease: a mathematical model with awareness programs and time delay," Discrete Dynamics in Nature and Society, vol. 2015, Article ID 260195, 13 pages, 2015.

[15] P. W. William, "Method for preventing and treating alcoholism: United States," Patent 06426368, 2002.

[16] X.-Y. Wang, H.-F. Huo, Q.-K. Kong, and W.-X. Shi, "Optimal control strategies in an alcoholism model," Abstract and Applied Analysis, vol. 2014, Article ID 954069, 18 pages, 2014.

[17] X.-Y. Wang, K. Hattaf, H.-F. Huo, and H. Xiang, "Stability analysis of a delayed social epidemics model with general contact rate and its optimal control," Journal of Industrial and Management Optimization, vol. 12, no. 4, pp. 1267-1285, 2016.

[18] Q. Yang, D. Jiang, N. Shi, and C. Ji, "The ergodicity and extinction of stochastically perturbed SIR and SEIR epidemic models with saturated incidence," Journal of Mathematical Analysis and Applications, vol. 388, no. 1, pp. 248-271, 2012.

[19] R. Z. Hasminskii, Stochastic Stability of Differential Equations, Kluwer Academic, Alphen aan den Rijn, The Netherlands, 1980.

[20] C. Zhu and G. Yin, "Asymptotic properties of hybrid diffusion systems," SIAM Journal on Control and Optimization, vol. 46, no. 4, pp. 1155-1179, 2007.

[21] P. van den Driessche and J. Watmough, "Reproduction numbers and sub-threshold endemic equilibria for compartmental models of disease transmission," Mathematical Biosciences, vol. 180, pp. 29-48, 2002.

[22] X. Mao, Stochastic Differential Equations and Applications, Horwood, Sawston, UK, 2007.

[23] D. J. Higham, "An algorithmic introduction to numerical simulation of stochastic differential equations," SIAM Review, vol. 43, no. 3, pp. 525-546, 2001. 


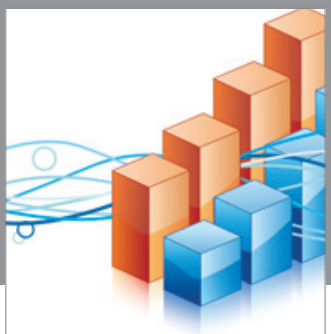

Advances in

Operations Research

vatem alat4

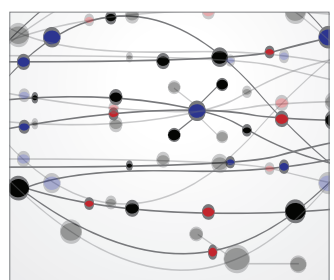

\section{The Scientific} World Journal
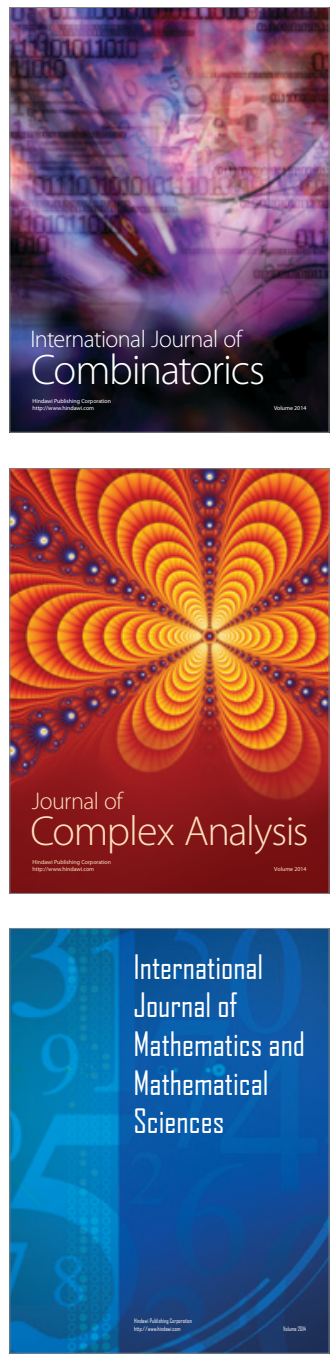
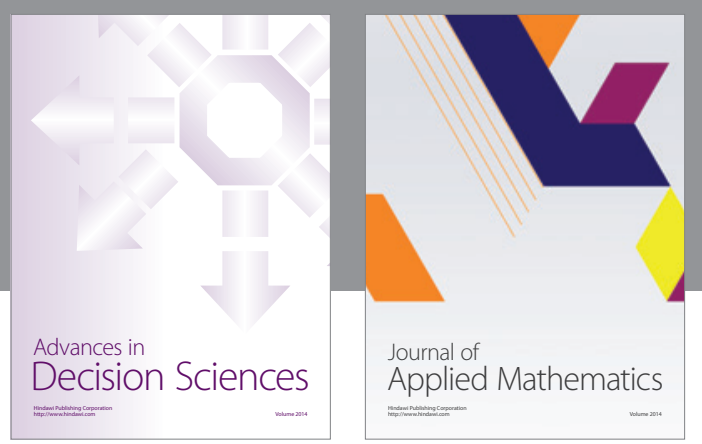

Algebra

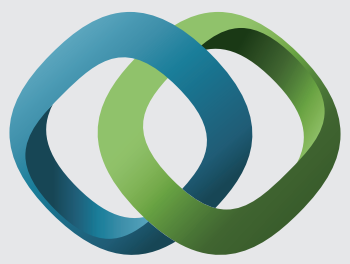

\section{Hindawi}

Submit your manuscripts at

https://www.hindawi.com
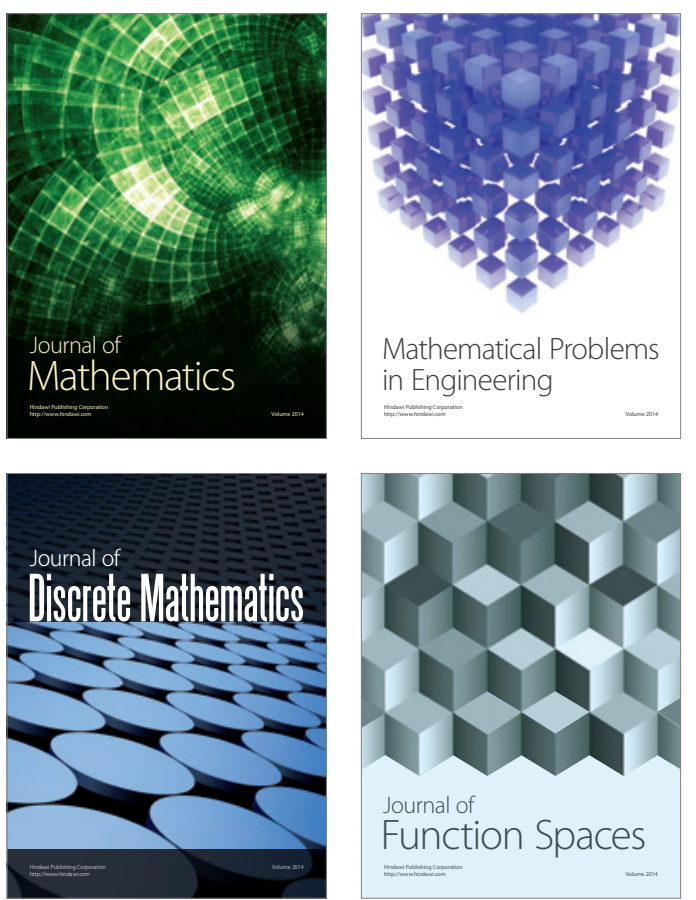

Mathematical Problems in Engineering
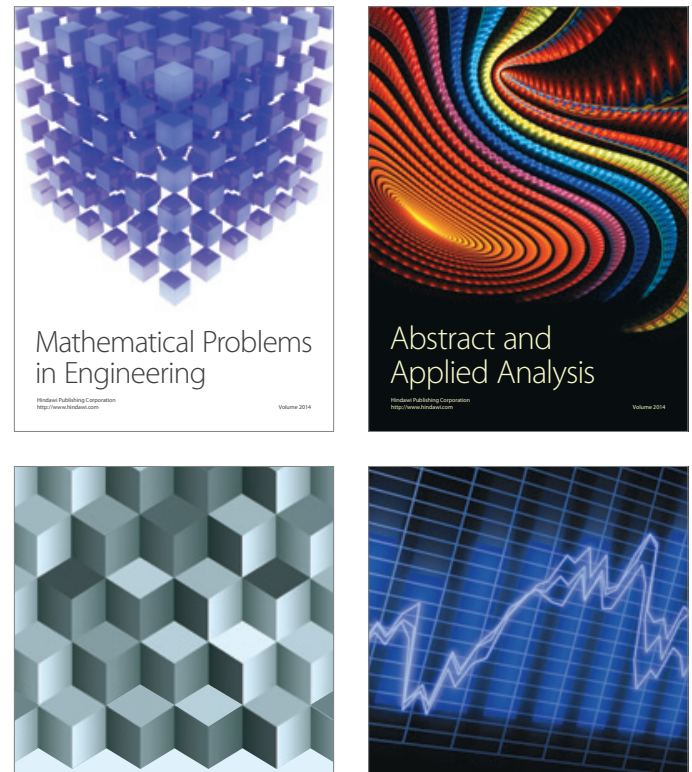

Journal of

Function Spaces

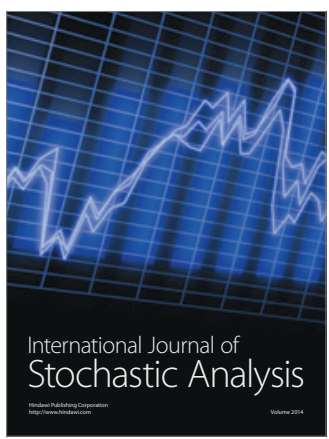

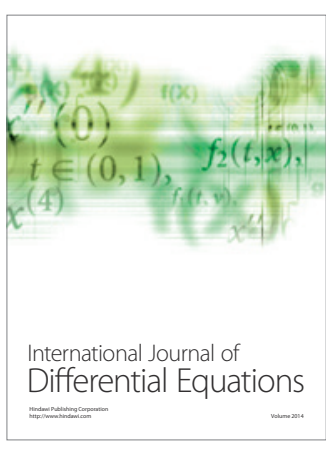
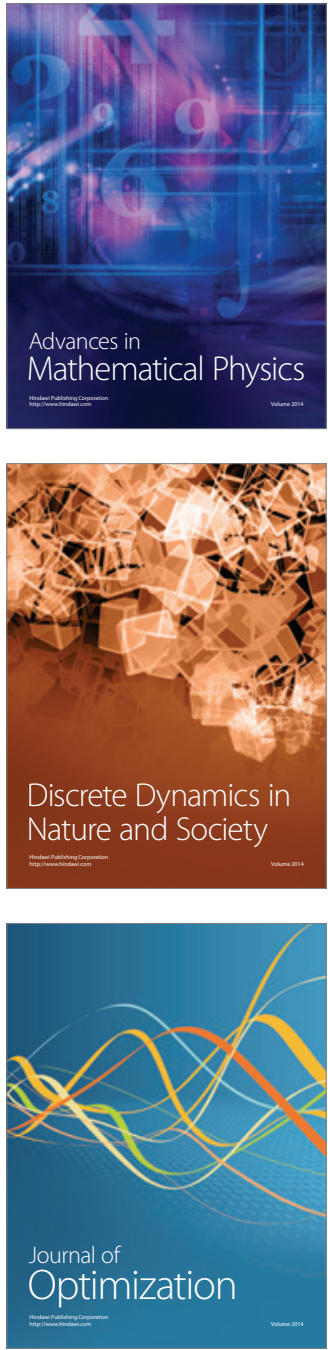